\title{
GATA4-dependent organ-specific endothelial differentiation controls liver development and embryonic hematopoiesis
}

Cyrill Géraud, ${ }^{1}$ Philipp-Sebastian Koch, ${ }^{1}$ Johanna Zierow, ${ }^{1}$ Kay Klapproth, ${ }^{2}$ Katrin Busch, ${ }^{2}$ Victor Olsavszky, ${ }^{1}$ Thomas Leibing, ${ }^{1}$ Alexandra Demory, ${ }^{1}$ Friederike Ulbrich, ${ }^{1}$ Miriam Diett, ${ }^{1}$ Sandhya Singh, ${ }^{1}$ Carsten Sticht, ${ }^{3}$ Katja Breitkopf-Heinlein, ${ }^{4}$ Karsten Richter, ${ }^{5}$ Sanna-Maria Karppinen, ${ }^{6}$ Taina Pihlajaniemi, ${ }^{6}$ Bernd Arnold, ${ }^{7}$ Hans-Reimer Rodewald, ${ }^{2}$ Hellmut C. Augustin, ${ }^{8,9}$ Kai Schledzewski, ${ }^{1}$ and Sergij Goerdt ${ }^{1}$

\begin{abstract}
'Department of Dermatology, Venereology, and Allergology, University Medical Center and Medical Faculty Mannheim, Heidelberg University, and Center of Excellence in Dermatology, Mannheim, Germany. ${ }^{2}$ Division of Cellular Immunology, German Cancer Research Center (DKFZ), Heidelberg, Germany. ${ }^{3}$ Center for Medical Research, ${ }^{4}$ Department of Medicine II, Section Molecular Hepatology, Medical Faculty Mannheim, Heidelberg University, Mannheim, Germany. ${ }^{5}$ Division of Molecular Genetics, DKFZ, Heidelberg, Germany. ${ }^{6}$ Faculty of Biochemistry and Molecular Medicine and Biocenter Oulu, University of Oulu, Oulu, Finland. 'Division of Molecular Immunology, DKFZ, Heidelberg, Germany. ${ }^{8}$ Division of Vascular Oncology and Metastasis (DKFZ-ZMBH Alliance), DKFZ, Heidelberg, Germany. ${ }^{9}$ Department of Vascular Biology and Tumor Angiogenesis (CBTM), Medical Faculty Mannheim, Heidelberg University, Mannheim, Germany.
\end{abstract}

Microvascular endothelial cells (ECs) are increasingly recognized as organ-specific gatekeepers of their microenvironment. Microvascular ECs instruct neighboring cells in their organ-specific vascular niches through angiocrine factors, which include secreted growth factors (angiokines), extracellular matrix molecules, and transmembrane proteins. However, the molecular regulators that drive organ-specific microvascular transcriptional programs and thereby regulate angiodiversity are largely elusive. In contrast to other ECs, which form a continuous cell layer, liver sinusoidal ECs (LSECs) constitute discontinuous, permeable microvessels. Here, we have shown that the transcription factor CATA4 controls murine LSEC specification and function. LSEC-restricted deletion of Gata4 caused transformation of discontinuous liver sinusoids into continuous capillaries. Capillarization was characterized by ectopic basement membrane deposition, formation of a continuous EC layer, and increased expression of VE-cadherin. Correspondingly, ectopic expression of CATA4 in cultured continuous ECs mediated the downregulation of continuous EC-associated transcripts and upregulation of LSEC-associated genes. The switch from discontinuous LSECs to continuous ECs during embryogenesis caused liver hypoplasia, fibrosis, and impaired colonization by hematopoietic progenitor cells, resulting in anemia and embryonic lethality. Thus, GATA4 acts as master regulator of hepatic microvascular specification and acquisition of organ-specific vascular competence, which are indispensable for liver development. The data also establish an essential role of the hepatic microvasculature in embryonic hematopoiesis.

\section{Introduction}

Endothelial cells (ECs) line the inside of all blood vessels, forming a structurally and functionally heterogeneous cell population $(1,2)$. The diversity of ECs along the segments of the vascular tree and in the microvascular beds of different organs has phenotypically long been recognized. It ranges from continuous, barrier-forming endothelium to discontinuous, fenestrated endothelia (3). The transcriptional programs regulating arterial, venous, and lymphatic specification have been characterized in substantial molecular detail in recent years. Delta/Notch signaling determines arterial differentiation (4), COUP-TFII controls venous cell fate (5), and PROX1 acts as a master regulator to induce lymphatic differentiation (6). In con-

Related Commentary: p. 790

Authorship note: C. Géraud, P.S. Koch, and J. Zierow contributed equally to this work. Conflict of interest: The authors have declared that no conflict of interest exists. Submitted: August 10, 2016; Accepted: December 20, 2016.

Reference information: J Clin Invest. 2017;127(3):1099-1114 https://doi.org/10.1172/JCI90086. trast, the molecular regulators driving organ-specific differentiation of microvascular ECs have not been elucidated.

Originally believed to form a rather inert, merely structure-building vascular cell population, microvascular ECs are now considered to actively control the tissue microenvironment (7). Primarily defined as paracrine-acting soluble growth factors, angiocrine factors are now more broadly recognized to comprise organ-specific signaling programs including extracellular matrix (ECM) molecules and cell surface proteins (7). These angiocrine factors control organogenesis during development as well as organ function during health and disease (8-12).

Among the microvascular beds of different organs, the hepatic sinusoids are a prime model of angiodiversity. In contrast to most other microvascular ECs, liver sinusoidal ECs (LSECs) are discontinuous, lack a basement membrane, show fenestrations, and exhibit specialized junctional complexes with a high permeability for solutes (13). They act as protective scavenger cells by clearing noxious blood factors from the circulation via endocytic receptors such as stabilin 1 (STAB1) and STAB2 (14). Recent evidence suggests that the hepatic microvasculature is subspecialized and 
A

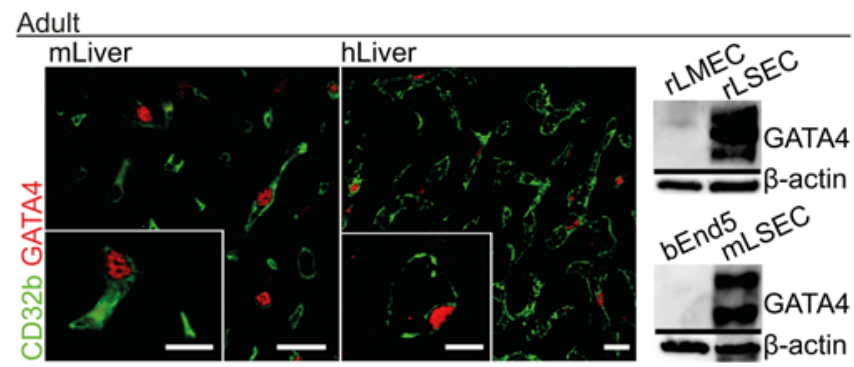

C

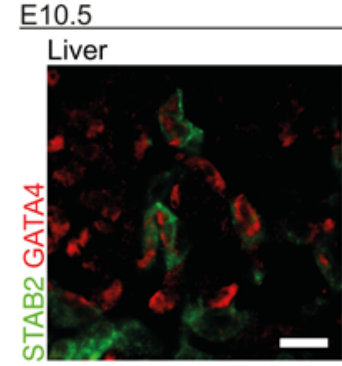

Heart
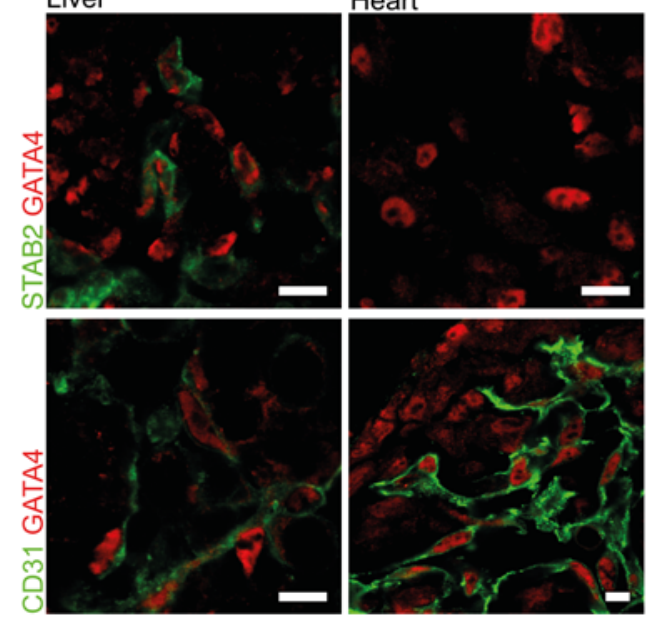

shows zonation along the liver sinusoids from periportal to centrilobular $(15,16)$. Furthermore, portal vein and central vein ECs may exert specialized functions required for hematopoiesis and liver zonation, respectively (17-19). Loss of organ-specific differentiation, i.e., transdifferentiation of the liver microvasculature, is involved in metabolic and cirrhotic liver diseases as well as liver cancer and metastases $(20,21)$.

It is well recognized that angiogenesis in general and organ development are coupled in the liver $(22,23)$ even prior to vascular function (24). The impact of LSEC-specific differentiation on organ development and function, however, and the superordinate molecular regulators involved are largely unknown. Elucidation of the molecular mechanisms that control organ-specific endothelial differentiation in the liver and their impact on cellular interactions during development will likely promote novel approaches to target liver diseases accompanied by endothelial transdifferentiation.

\section{Results}

Genetic inactivation of Gata4 in the hepatic microvasculature in vivo. Gata4 was previously identified by us in a cluster of transcription factors overexpressed in rat LSECs in comparison to rat lung microvascular ECs (rLMECs) (25). Nuclear GATA4 protein expression was detected in CD32 $\mathrm{b}^{+}$LSECs, but not in other cell types in adult murine and human liver tissue (Figure $1 \mathrm{~A}$ ) or in $\mathrm{CD} 31^{+}$microvascular ECs in other adult organs, such as human and murine heart (Supplemental Figure 1A; supplemental material available online with this article; https://doi.org/10.1172/JCI90086DS1). GATA4 expression was only observed in primary LSEC isolates of mice and rats, but not in rLMECs or in the murine brain EC line bEnd5
B

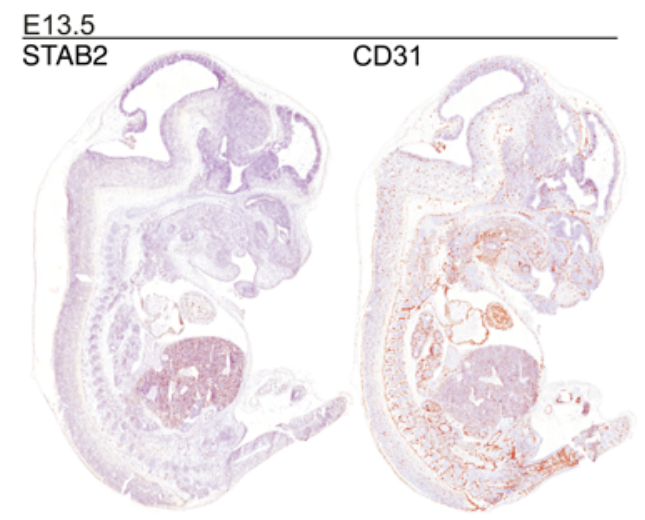

Figure 1. LSECs represent the only intersection of GATA4 and STAB2 expression during development of the embryo. (A) Murine liver (mLiver) and human liver (hLiver) show GATA4 in CD32b+ LSECs on co-immunofluorescence (co-IF). Expression of GATA4 detected by Western blot in rat LSECs (rLSEC) and mouse LSECs (mLSEC), but not in rLMECs and bEnd5 murine brain ECs $(n=3)$. Scale bars: $20 \mu \mathrm{m}$ and $10 \mu \mathrm{m}$ (inset). (B) IHC of STAB2 and CD31 at E13.5 $(n=5)$. (C) Co-IF of GATA4 with STAB2 or CD31 in fetal liver and heart of WT embryos at E10.5 $(n=2)$. Scale bars: $10 \mu \mathrm{m}$.

by Western blotting (Figure 1A). Therefore, GATA4 was a strong candidate for an LSEC-specific transcriptional regulator.

As GATA4 is a critical regulator of development in various cell types at different developmental stages, functional analysis of GATA4 in LSECs in vivo was hampered by the fact that general as well as pan-EC-targeted deficiency of Gata 4 caused early fetal lethality around E8.5 and E12.5, respectively. In the latter case, embryonic lethality was due to cardiac defects, precluding a detailed analysis of the functions of GATA4 in LSECs during development (26).

In order to delete GATA4 in LSECs, an EC-specific Cre deleter mouse was needed that would leave endocardial Gata4 expression intact. In contrast to established EC-specific Cre deleter mice in which Tie-2 or VE-cadherin promoters are utilized, STAB2 displayed a more restricted expression pattern, mostly confined to LSECs in the developing embryo; cardiac capillary ECs or endocardial ECs in ventricles or atria were not stained (Figure 1, B and C, and Supplemental Figure 1B). No PROX1/STAB2 double-positive ECs were identified in the liver at E11.5, indicating the lack of lymphatic EC differentiation in the liver at this developmental stage (Supplemental Figure 1D). Biliary associated blood vessels were identified as STAB2-CD31 $1^{+}$continuous blood vessels in adult liver (Supplemental Figure 2A). As expected, STAB2 ${ }^{+}$LSECs and DPPIV ${ }^{+}$biliary canaliculi were located on different sides of hepatocytes (Supplemental Figure 2A). Furthermore, STAB2 was expressed only by blood vascular ECs and not by other perivascular cells, such as macrophages or stellate cells (Supplemental Figure 1D). In addition, a comprehensive immunofluorescence screen of murine embryonic organs at E10.5 and E11.5 revealed that endothelial GATA4 expression was strictly confined to 
A

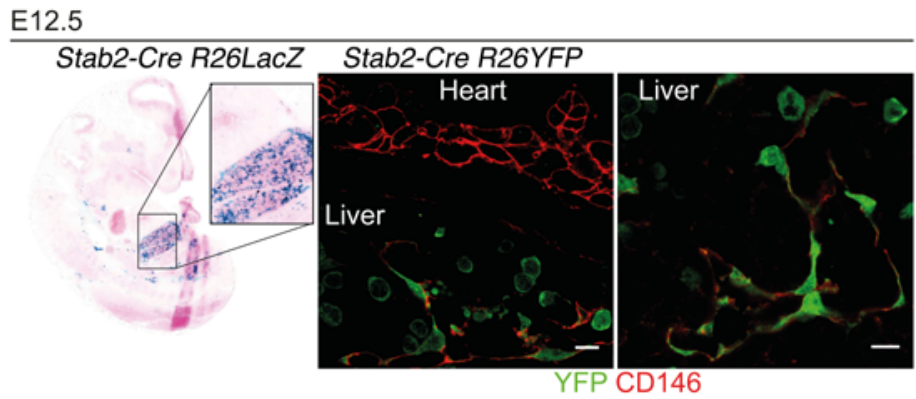

C

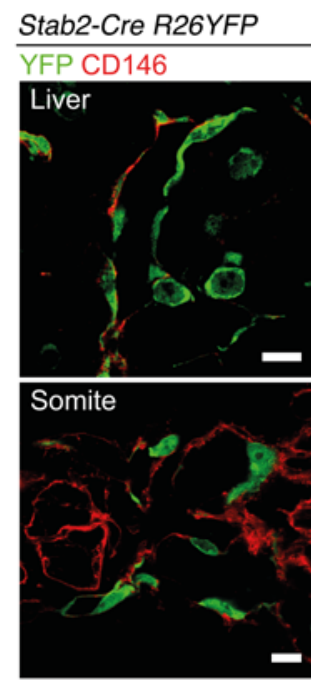

E

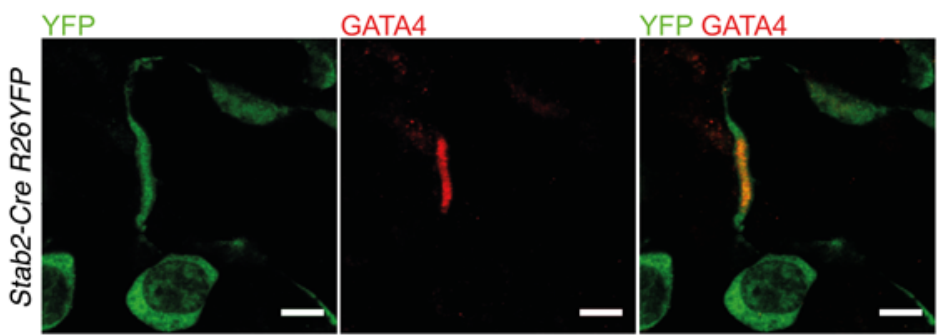

B
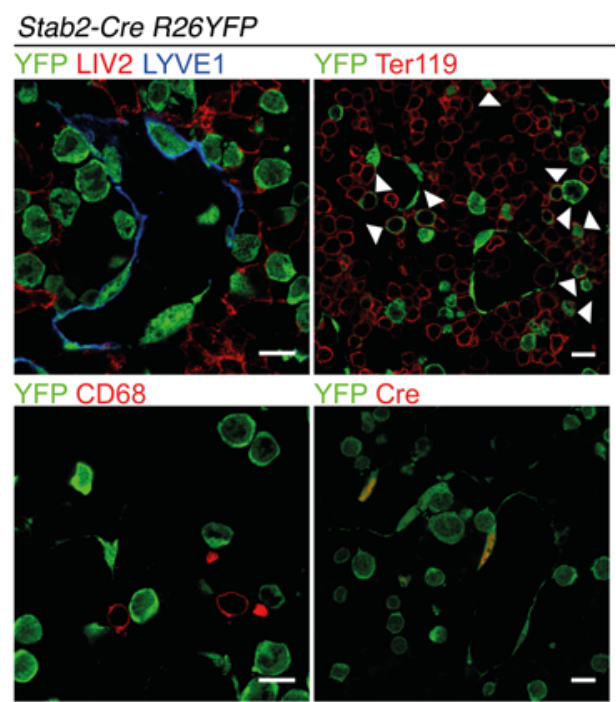

D

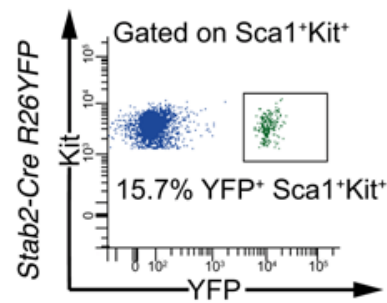

Figure 2. Characterization of endothelial subtype-specific Stab2-Cre driver mice. (A) Analysis of Stab2-Cre R26LacZ ( $\beta$-gal assay) ( $n=3$ ) and Stab2-Cre R26YFP (co-IF of YFP and CD146) $(n=3)$ shows reporter activity in the endothelium of the fetal liver. Scale bars: $10 \mu \mathrm{m}$. (B-E) Analysis of the Stab2-Cre R26YFP reporter in the embryo. (B) Co-IF of YFP and LYVE1, Cre, LIV2, Ter119, and CD68 in the fetal liver at E12.5. Arrowheads indicate YFP+Ter119+ cells $(n=3)$. Scale bars: $10 \mu \mathrm{m}$. (C) Co-IF of YFP and CD146 at E12.5 $(n=3)$. Scale bars: $10 \mu \mathrm{m}$. (D) FACS analysis of the fetal liver of Stab2-Cre R26YFP reporter mice at E13.25. Representative FACS blot showing YFP reporter activity in Sca1 ${ }^{+}$Kit $^{+}$cells $(n=5)$. (E) Co-IF of YFP and GATA4 in the fetal liver at E12.5 ( $\left.n=3\right)$. Scale bars: $5 \mu$ m.

CD31 ${ }^{\text {loSTAB2 }}{ }^{\text {hi }}$ LSECs in the fetal liver and to CD31 ${ }^{\text {hisTAB2 }}{ }^{-}$ECs in the endocardium, while broad GATA4 expression was detected in non-ECs, such as myocardium or hepatic mesenchyme (Figure $1 \mathrm{C}$ and Supplemental Figure 1C). Thus, overlapping expression of GATA4 and STAB2 was only present in liver endothelium.

Therefore, transgenic mice with Stab2 promoter-driven Cre expression were generated (Supplemental Figure 1E). In Stab2-Cre $R 26 \mathrm{LacZ}$ reporter mice, $\beta$-gal activity was predominantly found in LSECs at E12.5, while the endocardium as well as the fetal blood microvasculature of heart, lung, and brain were negative (Figure 2A). However, endothelium of larger blood vessels such as the dorsal aorta and the intersegmental vessels as well as the umbilical vessels showed weak reporter activity (Supplemental Figure 1F). Notably, activity of the Stab2-Cre R26LacZ reporter was lost from the dorsal aorta and the intersegmental vessels by E12.5, but not from LSECs. This may be explained by the finding that the endothelium of the primitive dorsal aorta derived from the splanchnic mesenchyme is replaced by ECs derived from somitic mesenchymal precursors in the definitive aorta and larger vessels (27). A detailed analysis of Stab2-Cre R26YFP reporter mice revealed that heterogeneous YFP expression occurred in a variable fraction of the blood vascular endothelium of most embryonic vascular beds at E12.5; in contrast, YFP reporter activity was not found in the endocardium (Figure 2, A and C). Similarly, PROX1/YFP double-positive cells were not identified in the fetal liver at E11.5 in Stab2-Cre R26YFP mice, further supporting lack of lymphatic ECs in the liver at this developmental stage (Supplemental Figure 4B). As expected, PROX1 was widely expressed in hepatocytes during liver development (Supplemental Figure 4B). When costaining YFP reporter activity with LIV2 (hepatoblasts) and 
A

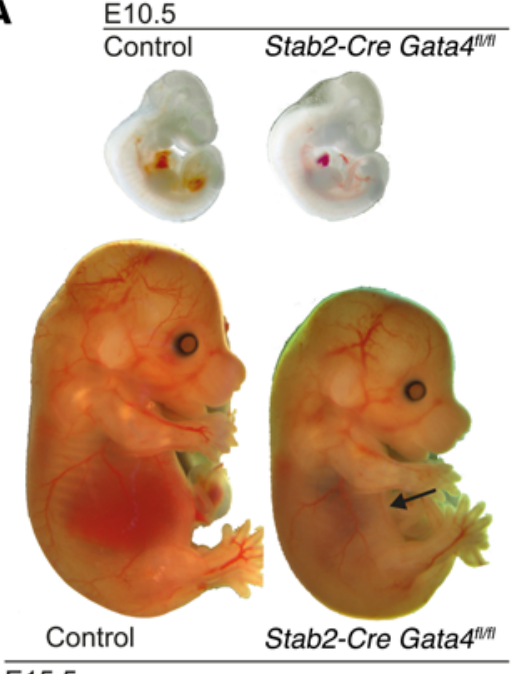

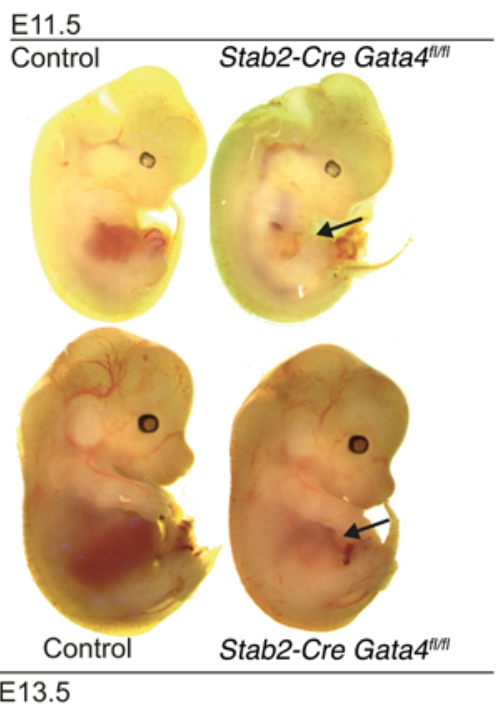

D $E 11.25$
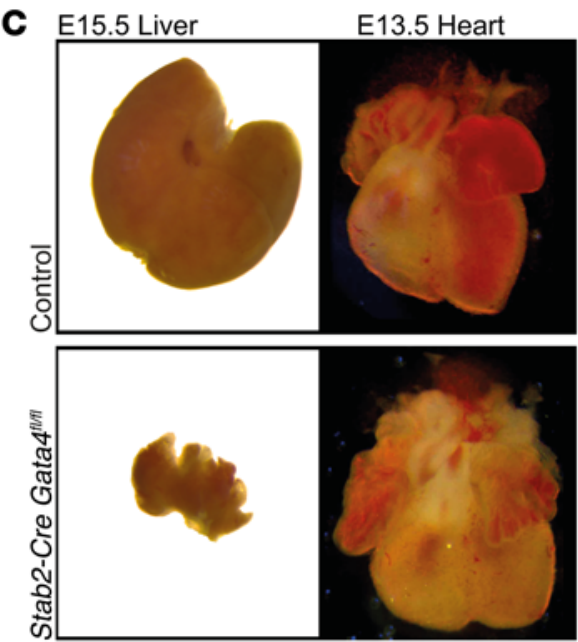

B

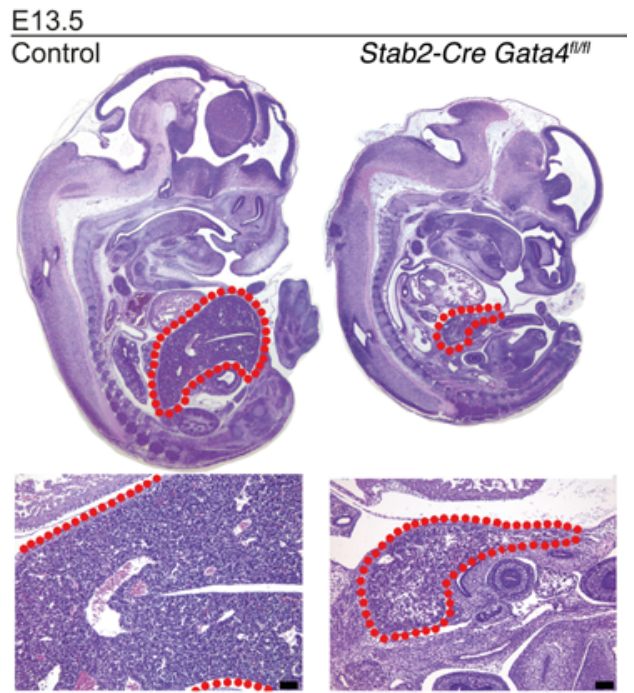

$\mathbf{E}$

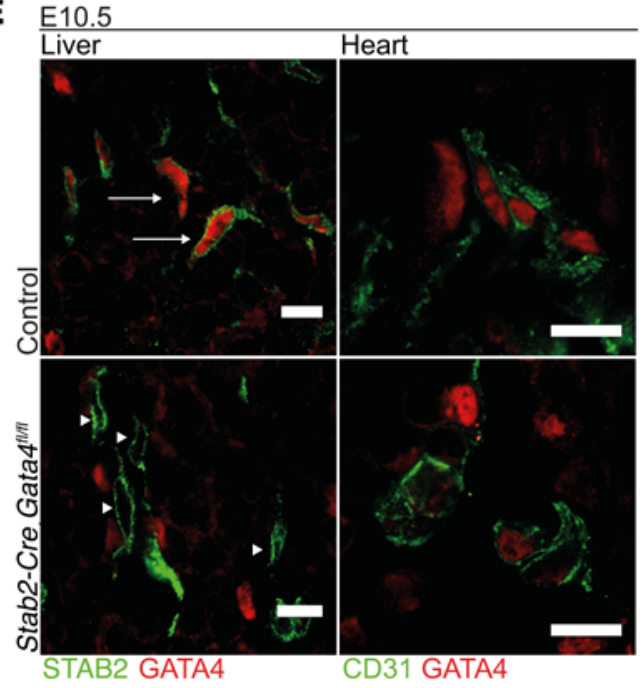

Figure 3. Deletion of GATA4 in LSECs in Stab2-Cre Gata4 ${ }^{f / f l}$ mice impairs liver development and causes embryonic lethality. (A) Photomicrographs of Stab2-Cre Gata4 $4^{f / f l}$ embryos at different ages. Arrows indicate hypoplastic livers in the mutant embryos from E11.5 to E15.5 ( $n>3$ ). (B) H\&E staining of Stab2-Cre Gata4fl/fl embryos at E13.5. Red dotted lines indicate the fetal liver ( $n=5)$. Scale bars: $100 \mu \mathrm{m}$. (C) Photomicrographs of the fetal liver (E15.5) and fetal heart (E13.5) of Stab2-Cre Gata4 fl/fl embryos. (D) FACS analysis of live cells, Ter119+ cells, and Ter119- cells in the liver of Stab2-Cre Gata $4^{f / / f l}$ embryos at E11.25 ( $n=14$ mutants and 10 controls). Student's $t$ test; ${ }^{*} P<0.05$, ${ }^{* * *} P<0.001$. (E) Co-IF of GATA4 and STAB2 or CD31 of fetal livers of Stab2-Cre Gata4f//fl embryos at E10.5. IF shows absence of GATA4 in the mutant liver (arrowheads), but not in controls (arrows) $(n=3)$. Scale bars: $10 \mu \mathrm{m}$.

CD68 (macrophages) in Stab2-Cre R26YFP mice, no double labeling was detected (Figure 2B). Stab2-Cre R26YFP reporter activity was also detected in CD146- (Figure 2C), LYVE1- (Figure 2B), and CD31- (Supplemental Figure 4B) non-ECs, with a distinct round morphology in the fetal liver. Of the $\mathrm{YFP}^{+}$cells, a small percentage ( 10\%) were $\mathrm{Kit}^{+}$hematopoietic stem (HSCs) and progenitor cells (Supplemental Figure 3A). Conversely, approximately 16\% of the Sca $1^{+} \mathrm{Kit}^{+} \mathrm{HSCs}$ and progenitor cells were $\mathrm{YFP}^{+}$(Figure 2D). In addition, YFP expression was also detected in approximately $25 \%$ of $\mathrm{CD}^{4} 5^{+} \mathrm{Sca}^{-} \mathrm{Kit}^{-}$hematopoietic cells (Supplemental Figure $3 \mathrm{~A})$. Few of the $\mathrm{YFP}^{+}$"round" cells were Ter119+ erythrocyte precursors, but most did not express Ter119 (Figure 2B). Notably, the $\mathrm{YFP}^{+}$round cells did not show any STAB2, GATA4, or Cre protein expression at E11.5 or E12.5 (Figure 2, B and E; Supplemental
Figure 3, B and C; and Supplemental Figure 4B). As other organs harboring $\mathrm{STAB}^{+}$sinusoidal vessels such as spleen, lymph node, and bone marrow are not developed at E10.5/E11.5 and as STAB2 ${ }^{+}$ sinusoidal ECs in these organs in adult mice do not normally express GATA4 (Supplemental Figure 2B), these data demonstrate that LSECs present the only intersection of GATA4 expression and Stab2 promoter activity during development of the embryo.

Similar to STAB2, LYVE1 shows restricted vascular expression in LSECs, but LYVE1 is also known to be expressed by lymphatic endothelium as well as by HSCs and progenitor cells and by some macrophage populations (28). In Lyve1-Cre R26LacZ reporter mice, $\beta$-gal activity was predominantly found in LSECs (Supplemental Figure 5A). When analyzing Lyve1-Cre R26YFP reporter mice, we found that distribution of Lyve1 reporter activity in the 


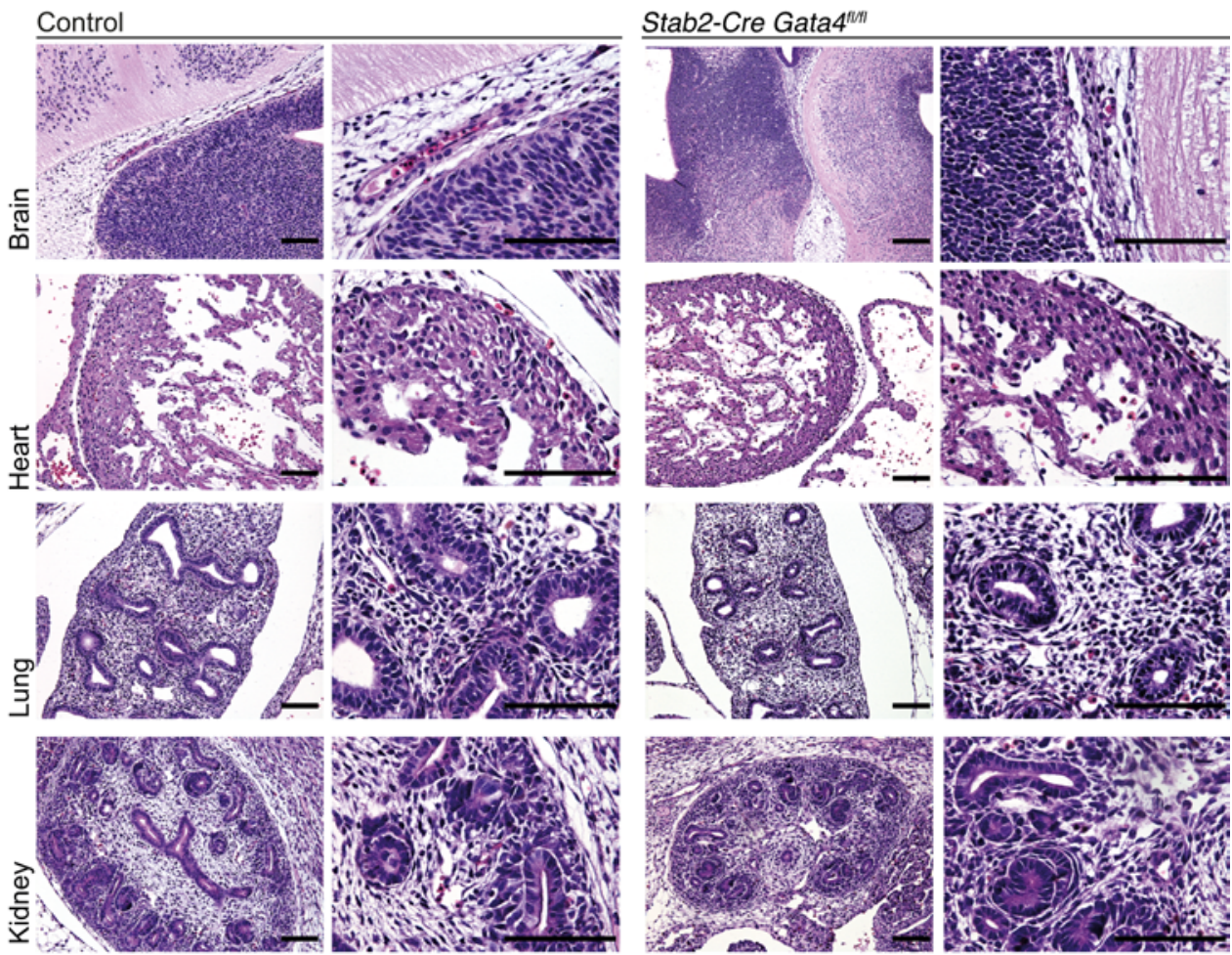

Figure 4. Deletion of Gata4 in LSECs in Stab2-Cre Gata4 $4^{f / f l}$ mice does not affect cardiac, brain, lung, or kidney development. H\&E staining of the brain, heart, lung, and kidney of Stab2-Cre Gata $f^{f / / f l}$ embryos at E13.5 does not show any gross abnormalities $(n=3)$. Scale bars: $100 \mu \mathrm{m}$.

ative non-ECs, which likely correspond to mesenchymal cells that originate from the septum transversum mesenchyme and are well known to express GATA4 (29).

Lyve1-Cre Gata4 ${ }^{f l f l}$ mutant embryos had a phenotype that was similar to albeit slightly less severe than that of Stab2-Cre Gata4 $4^{f / f l}$ embryos. This may be due to the fact that the onset of activity of the Lyve1 promoter is delayed by 1 embryonic day as compared with the Stab2 promoter (30). Nevertheless, no Lyve1-Cre Gata $4^{f / f l}$ mice were born alive among a total of 48 pups (Supplemental Figure 5C). Macroscopic and histologic examination at different embryonic stages revealed a hypoplastic liver in Lyve1-Cre Gata $4^{f / f l}$ mice, while the heart and all other organs were normal in size and structure (Figure 5, A and B). Furthermore, the reduction in the absolute numbers of live cells in dissected Lyve1-Cre Gata $^{f l / f l}$ fetal livers was slightly less severe than in Stab2-Cre Gata4 ${ }^{\text {flfl }}$ embryos (Figure 5D). The specific-

fetal liver at E12.5 was similar to that already demonstrated in Stab2-Cre R26YFP reporter mice (Supplemental Figure 5B).

Together, these findings strongly indicate that the combination of either Stab2 or Lyve1 promoter-driven Cre transgenes with Gata $^{f / f l}$ mice allows specific genetic inactivation of Gata4 in liver ECs during development.

GATA4 expression in LSECs controls liver development. To address GATA4 functions in the liver microvasculature, we pursued homozygous deletion of Gata4 in LSEC by crossing Gata $4^{f / f l}$ mice with Stab2-Cre and Lyve1-Cre deleter mice. Among a total of 149 pups, no Stab2-Cre Gata $4^{f l / f l}$ mice were born alive. Stab2-Cre $\mathrm{Gata}^{f l / f l}$ embryos were found at the expected Mendelian frequency and showed lethality between E15.5 and E17.5 (Supplemental Figure 1G). Macroscopic and histologic examination at different embryonic stages revealed a severely hypoplastic liver starting at E11.5 in Stab2-Cre Gata $4^{f l / f l}$ embryos, while the heart and all other organs were unaltered in size and structure (Figure $3, \mathrm{~A}^{-} \mathrm{C}$, and Figure 4). Furthermore, FACS analysis showed a major reduction in the absolute numbers of live cells, as well as of Ter119+ ${ }^{+}$erythroid cells, and of Ter119- cells in dissected Stab2-Cre Gata $4^{f / f l}$ fetal livers (Figure 3D). The specificity of the deletion of Gata4 in LSECs was confirmed by immunofluorescence analysis for GATA4 in mutant and control embryos. GATA4 expression was deleted in $\mathrm{STAB}^{+}$ECs in Stab2-Cre Gata ${ }^{f / f l}$ livers, with a knockdown efficiency of approximately $90 \%$ (Figure 3E and Supplemental Figure 3D), and the lack of GATA4 in liver ECs was the only difference in GATA4 expression between control and mutant embryos (Figure $3 \mathrm{E})$. In contrast, GATA4 expression was preserved in STAB2-neg- ity of the deletion of GATA4 in LSECs in Lyve1-Cre Gata $4^{f / f l}$ mice was confirmed by immunofluorescence analysis for GATA4 in mutant and control embryos (Figure 5C). Collectively, these data show that liver sinusoidal endothelial GATA4 is an indispensable molecular regulator of fetal development.

GATA4 promotes discontinuous differentiation of LSECs. To study the role of GATA4 in EC structure and differentiation, we assessed quantitative and qualitative parameters of the liver microvasculature. The expression of LSEC-associated proteins STAB2 and LYVE1 was considerably downregulated in the livers of Stab2-Cre Gata $4^{f / f l}$ and Lyve1-Cre Gata $4^{f l / f l}$ embryos, while CD31 was upregulated (Figure 6, A and C), indicating a major switch from a sinusoidal $\left(\mathrm{LYVE} 1^{\text {hi }} \mathrm{STAB} 2^{\mathrm{hi}} \mathrm{CD} 31^{\text {lo }}\right)$ to a continuous capillary phenotype $\left(\mathrm{LYVE}^{-} \mathrm{STAB}{ }^{-} \mathrm{CD} 31^{\mathrm{hi}}\right)$ within the hepatic microvasculature. Capillarization was seen at E11.5 (Figure 6, A-C), but also at E10.5 (Figure 6D), clearly before the livers became hypoplastic. Despite capillarization, the overall hepatic vascular density was only slightly reduced in the livers of Stab2-Cre Gata $4^{f / f l}$ embryos (Supplemental Figure 6C). Electron microscopy showed formation of a subendothelial basement membrane in hepatic microvessels of Stab2-Cre Gata $4^{f / f l}$ mice (Figure 6B and Supplemental Figure $1 \mathrm{H}$ ), confirming the switch from discontinuous sinusoids to continuous capillaries on the ultrastructural level.

To discriminate between transdifferentiation of LSECs after Gata4 deletion and replacement of these LSECs by Gata4 recombination-negative preexistent continuous ECs, we generated Stab2-Cre R26YFP Gata $4^{f / f l}$ triple mutant mice for lineage tracing. In this mouse model, the emerging continuous ECs would only be YFP reporter 
A

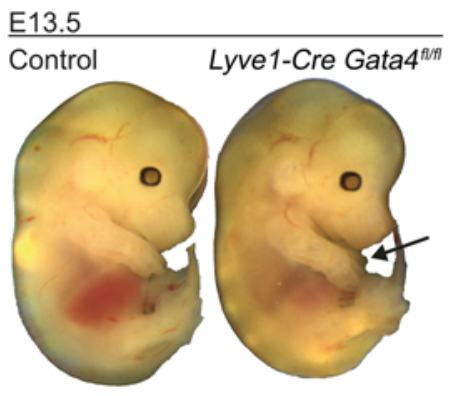

D

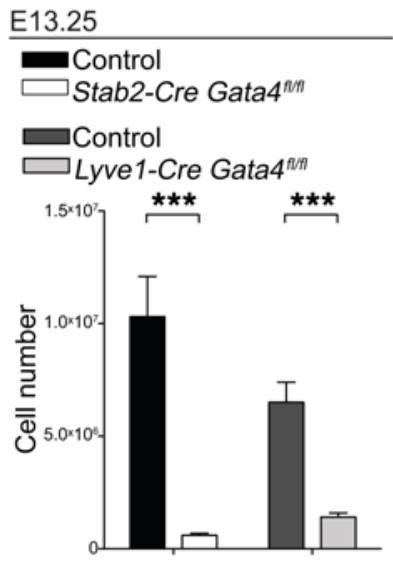

B

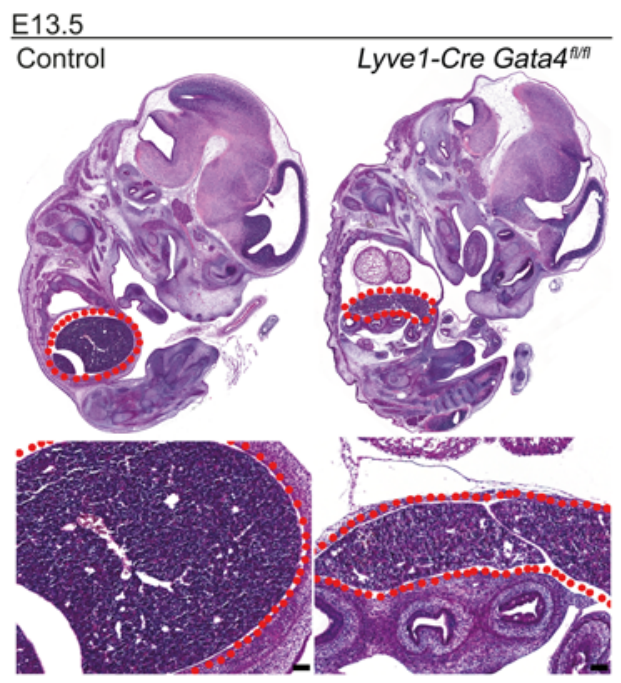

C

E11.5

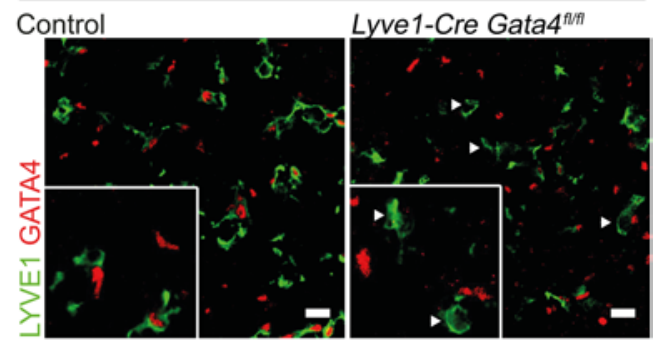

Figure 5. Lyve1-Cre Gata $4^{f / f l}$ mice show liver hypoplasia and embryonic lethality similar to but less severe than that observed in Stab2-Cre Gata4 ${ }^{f / f l}$ mice. (A and B) Photomicrographs (A) and H\&E staining (B) of Lyve1Cre Gata $4^{f / f l}$ embryos at E13.5. Arrow indicates the hypoplastic fetal liver, and red dotted lines indicate the fetal liver $(n=3)$. Scale bars: $100 \mu \mathrm{m}$. (C) Co-IF of GATA4 and LYVE1 of fetal livers of Lyve1-Cre Gata ${ }^{f / f f}$ embryos at E11.5. IF shows absence of GATA4 in the mutant liver (arrowheads), but not in controls $(n=3)$. Scale bars: $10 \mu \mathrm{m}$. (D) Total cell numbers of the liver of Stab2-Cre Gata4 ${ }^{f / f l}$ and Lyve1-Cre Gata4 ${ }^{f / f l}$ embryos at E13.25 (Stab2-Cre Cata $^{f / f \mid f l}: n=13$; Lyve1-Cre Gata4fl/fl: $n=6$ ). Student's $t$ test; ${ }^{* *} P<0.001$.

positive when they had Cre expression history, as in STAB2-expressing LSECs. In Gata4-deficient Stab2-Cre R26YFP reporter mice, CD $31^{\text {hi }}$ continuous ECs were generally $\mathrm{YFP}^{+}$, as were $\mathrm{LYVE1}{ }^{\text {hi }}$ and STAB2 ${ }^{\text {hi }}$ bona fide LSEC precursors (Supplemental Figure 4, A and B). Hence, CD $31^{\text {hi }}$ continuous ECs in mutant livers are derived from formerly STAB2 ${ }^{+}$ECs that have undergone Gata4 recombination.

GATA4 acts as a molecular master regulator of organ-specific angiodiversity. Gene expression analyses were performed on Gata4-deficient mutant mouse livers of Stab2-Cre Gata $4^{f / / f l}$ mice as compared with control embryos (Supplemental Figure 7). Primary mouse and rat LSECs rapidly dedifferentiate in culture (Supplemental Figure 6, D and E), precluding knockdown experiments. Therefore, human GATA4-transgenic HUVECs (HUVEC-GATA4) were generated to compare GATA4-mediated gene expression changes in this cell line (Supplemental Figure 6, $\mathrm{F}-\mathrm{H}$ ) with our findings in the fetal liver and with existing data (25) comparing rat LSECs and rat LMECs (Figure 7A). The results of these gene expression analyses clearly show that GATA4 strongly repressed expression of continuous EC-associated genes. In GATA4-transgenic HUVECs, 21 of 24 continuous EC-associated genes $(87.5 \%)$ were directly downregulated by GATA4 expression; of these 21 continuous EC-associated genes, 18 (85.7\%) were significantly upregulated by Gata 4 deficiency in mutant mouse livers (Cd31, Cav, Lama4, Fgfr1, Meox2, Emcn, Acvrl1, Sox18, Nr2f2, Angptl2, Hes1, Rgs5, Rgs4, Stc1, Plod2, Gabre, CD34, Apln) (Figure 7, A, C, and D, and Supplemental Figure 8B). Twelve of these genes (66.6\%) were also downregulated in rat LSECs as compared with rat LMECs. These results represent an unexpectedly high degree of concordance across species and different types of ECs. In con- trast, regulation of LSEC-associated genes by Gata4 manipulation was less stringent (Figure 7, A and B, and Supplemental Figure 8A).

In addition, gene expression profiling of Gata4-transgenic brain endothelioma-derived endothelial-like cell line bEnd3 was performed in order to analyze a continuous EC subtype from the opposite end of the spectrum of endothelial differentiation as compared with LSECs. Even in these ECs, Gata4 overexpression induced several LSEC-associated genes and suppressed expression of several continuous EC-associated genes (Supplemental Figure 10). LSEC-specific gene regulation by GATA4 in vitro was context dependent also in a broader sense, i.e., GATA4-dependent EC-associated genes were not induced by GATA4 transduction of a non-endothelial smooth muscle cell line; conversely, GATA4-dependent myocardial genes were not induced by GATA4 transduction of EC lines (Supplemental Figure 8, C and D).

A published list of GATA4 target genes in murine liver based on GATA4 chromatin immunoprecipitation followed by high-throughput sequencing (ChIP-seq) (31) was aligned with expression profiling data obtained from Stab2-Cre Gata $4^{f / f l}$ livers in order to delineate direct transcriptional targets of GATA4. Of those genes grouped into the endothelial gene ontology clusters, 21 of 44 (47\%) were directly regulated by GATA4 (Supplemental Figure 7), while only 21 of 172 (12\%) of those genes grouped into other gene ontology clusters were directly regulated by GATA4. These data indicate that a high percentage of EC-specific genes is directly regulated by GATA4 binding, while this is not the case for other cell types.

In Lyve1-Cre Gata4 $4^{f / f l}$ mutant livers, counterregulation of LSEC-associated genes and of continuous EC-associated genes was confirmed upon immunofluorescence analysis (Figure 6C, 
A

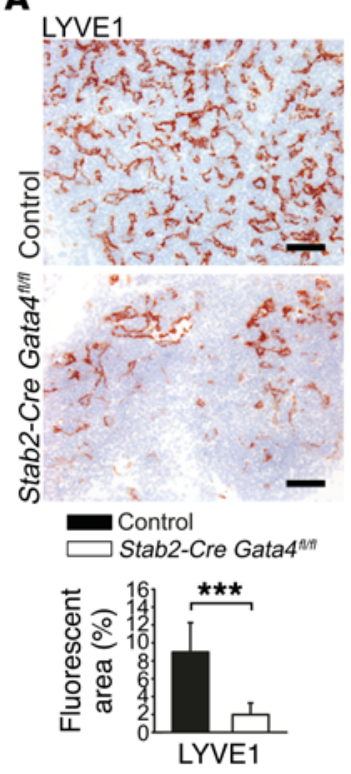

C
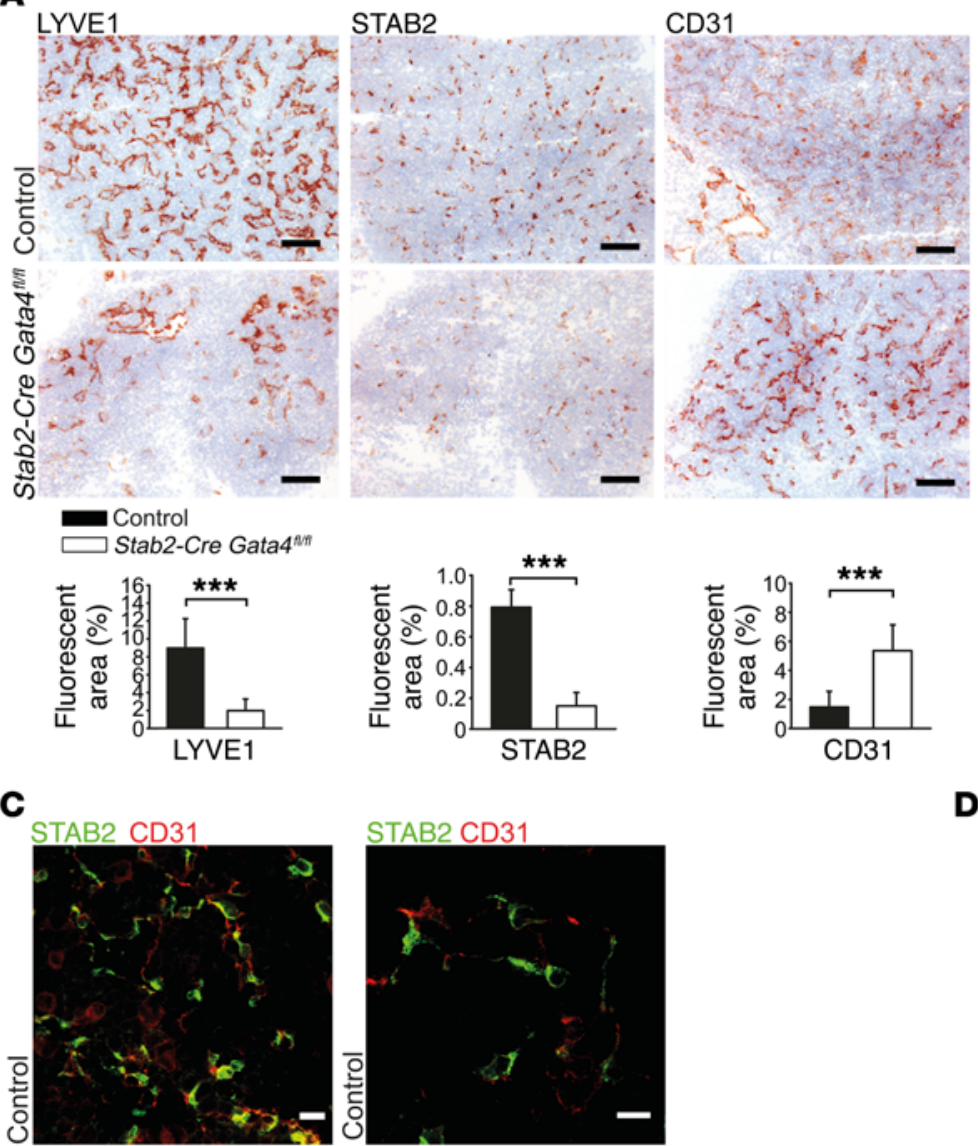

B

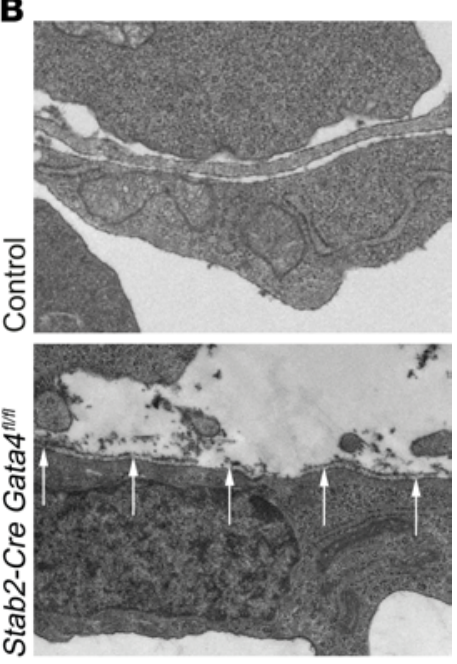

D $\underline{E} 10.5$
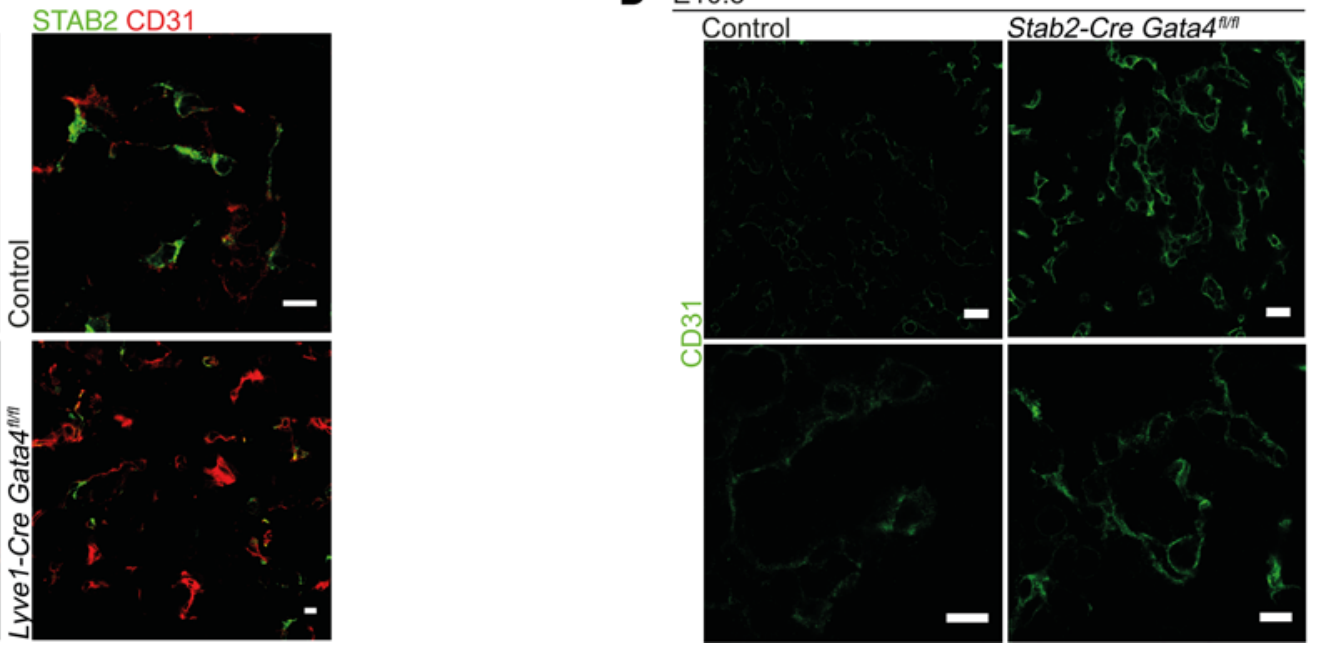

Figure 6. Liver endothelial GATA4 is required for maintenance of discontinuous LSEC differentiation. (A) Expression of LYVE1, STAB2, and CD31 in the liver of Stab2-Cre Gata4 fl/fl embryos at E11.5. IHC (upper panels) and quantification (lower panels) based on IF images of LYVE1, STAB2, and CD31 ( $n=4$ ). Scale bars: $100 \mu \mathrm{m}$. Western blot of LYVE1 and GAPDH with quantification of densitometric data $(n=5)$ (right). (B) Transmission electron microscopy (TEM) of the liver of Stab2-Cre Gata4f/ffl embryos at E11.5. Arrows indicate formation of a basement membrane $(n=4)$. (C) Co-IF of STAB2 and CD31 in the liver of Stab2-Cre Gata4fl/fl $(n=4)$ (left) and Lyve1-Cre Gata fl/ff $(n=3)$ (right) embryos at E11.5. Scale bars: $20 \mu \mathrm{m}$ (left) and $10 \mu \mathrm{m}$ (right). (D) IF of CD31 in the fetal liver of Stab2-Cre Gata $4^{f / f l}$ embryos at E10.5 shows increased expression of CD31 in the endothelium of mutant embryos as an indicator of early hepatic capillarization. Scale bars: $20 \mu \mathrm{m}$ (upper panels) and $10 \mu \mathrm{m}$ (lower panels) $(n=2)$. Student's $t$ test; ${ }^{* *} P<0.001$.

Figure 8A, and Supplemental Figure 5D). Together, our findings of counterregulation of discontinuous LSEC-associated versus continuous EC-associated genes in vivo and in vitro provide broad evidence that transcription factor GATA4 acts as a key regulator of LSEC differentiation and thereby hepatic angiodiversity.

Liver endothelial-related angiokine-deficient mice do not phenocopy liver endothelial Gata4 deficiency. As one of the hallmarks of instructive functions of ECs in tissue regeneration is secretion of angiokines from organ-specific ECs (7), we reasoned that LSEC-specific angiokines could cause the pathology in liver endothelial Gata4-deficient mice. Among the proteins induced in HUVEC-GATA4, BMP2 and RSPO3 were identified as GATA4-dependent angiokines (Figure 7B and Supplemental Figure 8, A and E). In addition, primary murine LSECs quickly dedifferentiated in culture, including downregulation of Gata4 as well as of LSEC-derived angiokines Bmp2, Rspo3, Wnt2, and $H g f$ (Supplemental Figure 6E). In contrast, these LSEC-derived angiokines as well as Ang2 were not significantly downregulated in Stab2-Cre Gata4 $4^{f / f l}$ fetal livers, as shown by gene expression profiling. Although it thus seemed unlikely that one of these angiokines might cause the developmental defects in Stab2-Cre Gata4 $^{f l / f l}$ mice, Stab2-Cre Bmp2 $2^{f / f l}$, Stab2-Cre Hgfl/fl, Stab2-Cre Rspo3 ${ }^{f l / f l}$, and Stab2-Cre Wlst/fl (deficient in wntless homology/ Wnt ligand secretion mediator with abolished secretion of all 
A

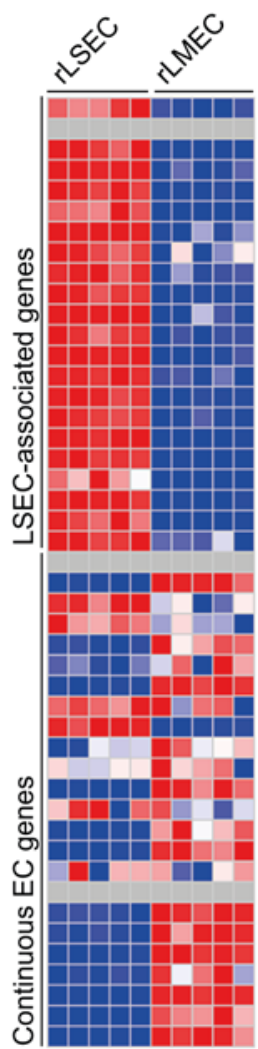

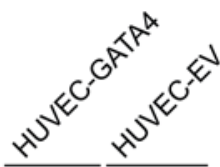

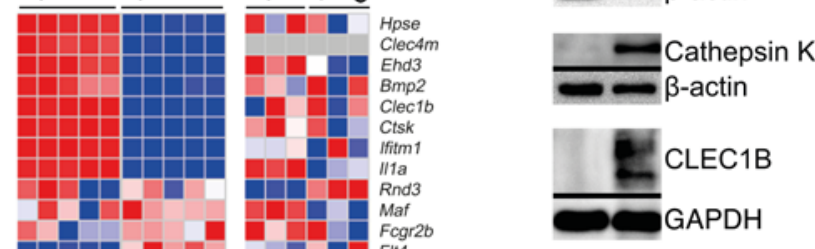

B

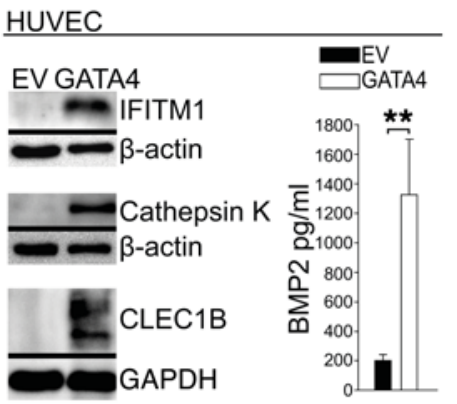

C HUVEC

\section{EV}

$\square$ GATA4

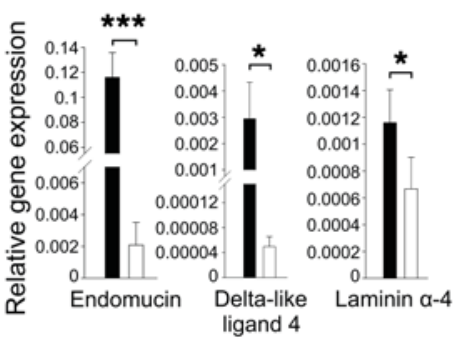

D
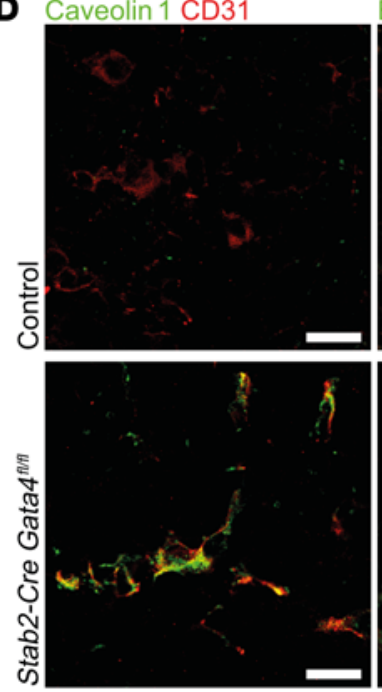

Endomucin CD31
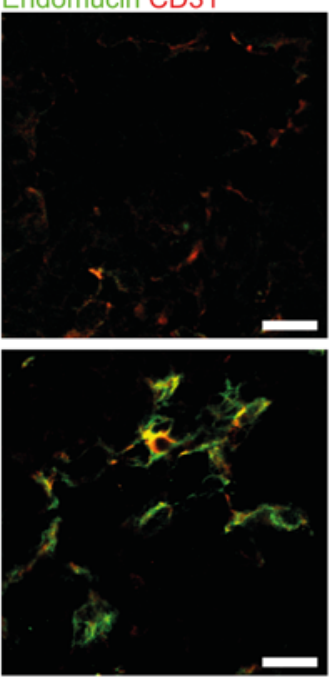

Figure 7. Endothelial GATA4 counterregulates liver sinusoidal and continuous EC differentiation in vivo and in vitro. (A) Heatmap of LSEC-associated and continuous EC-associated genes comparing primary rLSECs with rLMECs, GATA4-transduced with EV-transduced HUVECs, and liver from Stab2-Cre Gata4fl/fl embryos with control embryos at E11.5. (B) Western blot of IFITM1, cathepsin K, and CLEC1B of GATA4-transduced HUVECs ( $n=3$ ) (left). ELISA of BMP2 from supernatants of GATA4-transduced HUVECs $(n=3)$ (right). (C) qRT-PCR of endomucin, delta-like ligand 4, and laminin $\alpha 4$ in GATA4-transduced HUVECs $(n=3)$. (D) Co-IF of CD31 with caveolin 1 or endomucin in the liver of Stab2-Cre Gata4 ${ }^{f / f l}$ embryos at E11.5 ( $\left.n=4\right)$. Scale bars: $20 \mu m$. Student's $t$ test; ${ }^{*} P<0.05,{ }^{* *} P<0.01,{ }^{* *} P<0.001$.

Wnt factors by LSECs) mice were generated to assess the functional role of LSEC-derived angiokines during liver development. While Stab2-Cre Bmp $2^{f / f l}$, Stab2-Cre Hgfl/fl, and Stab2-Cre $W l^{f f / f l}$ mice survived until adulthood without showing any gross abnormalities, Stab2-Cre Rspo $3^{f / f l}$ mice died in utero in the late fetal period. Nevertheless, none of these LSEC-derived angiokine-deficient mice including Stab2-Cre Rspo $3^{\text {t/fl}}$ mice showed gross defects of liver development similar to Stab2-Cre Gata $4^{\text {//fl }}$ or Lyve1-Cre Gata ${ }^{\sharp / / l}$ mice at E12.5-E15.5 (Supplemental Figure 9, A-E). In addition, immunohistochemical analyses with vascular markers did not show capillarization of LSECs or defects of hematopoiesis in these mutant livers at E12.5 (Supplemental Figure 9, B-E). It has been reported recently that inducible endothelial Wls-deficient adult mice and inducible global Rspo3-deficient mice show impaired liver zonation $(18,19)$. As liver zonation becomes functional only after postnatal day 30 , these results do not contradict our findings. Together, these data indicate that the observed hepatic capillarization and liver hypoplasia in Stab2-Cre Gata $4^{f / f l}$ or Lyve1-Cre Gata $4^{A / f l}$ embryos are not a result of altered secretion of known angiokines by LSECs.
GATA4 controls junctional stability of LSECs and perivascular ECM deposition. As adherens junctional complexes that regulate vascular permeability differ between LSECs and continuous ECs (13), we analyzed VE-cadherin protein expression. IHC of E11.5 fetal liver sections of Stab2-Cre Gata4 $4^{f / f l}$ as well as of Lyve1-Cre Gata $4^{f / f l}$ mice demonstrated increased expression of VE-cadherin in the endothelium (Figure 8A). In HUVEC-GATA4, VE-cadherin and $\beta$-catenin were downregulated in comparison to HUVECs transduced with empty vector (HUVEC-EV) (Figure 8, B and C). In addition, co-immunoprecipitation experiments demonstrated reduced complex formation between VE-cadherin and $\beta$-catenin in HUVEC-GATA4 (Figure 8D). These findings indicate increased stability of adherens junctions in GATA4-deficient ECs in the capillarized hepatic microvessels in mutant livers.

Capillarization of hepatic sinusoids not only precedes, but is also permissive for the development of liver fibrosis $(20,21)$. As LSEC-restricted Gata4 deficiency was associated with capillarization of the hepatic sinusoids and with deposition of a basement membrane, this prompted us to analyze the ECM in Stab2-Cre Gata $^{f / f l}$ mice. Sirius red-positive collagen fibers were markedly 
A $\mathrm{E} 11.5$

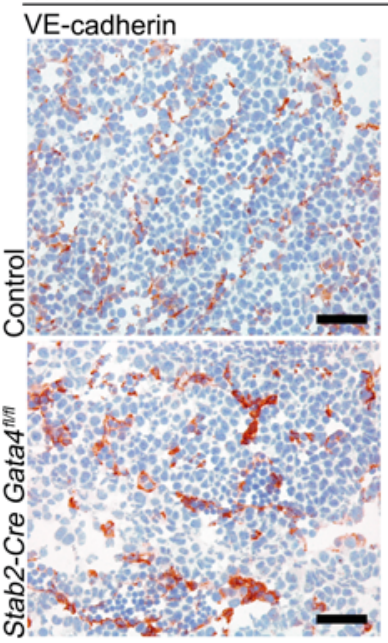

C
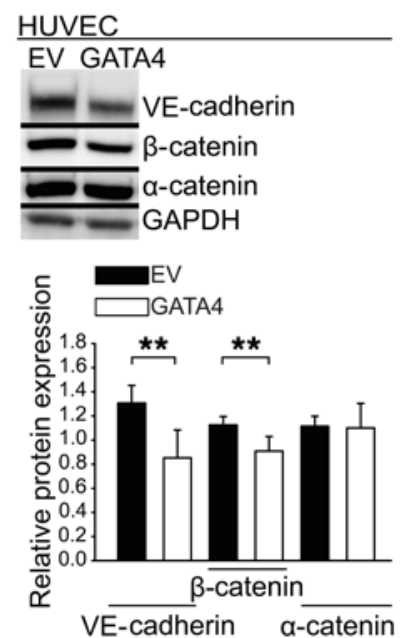
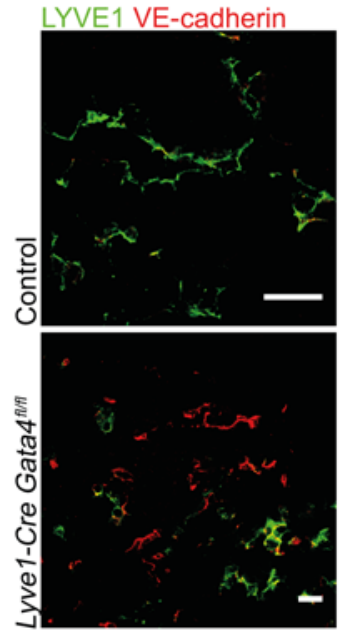

B HUVEC

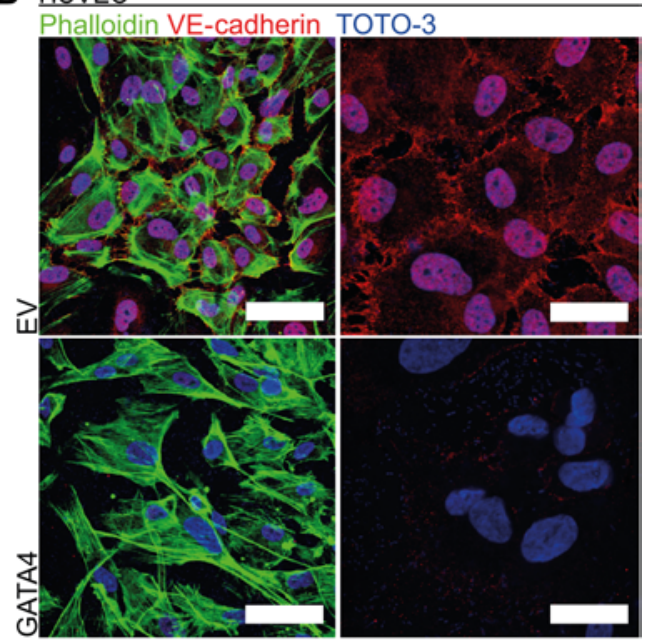

D

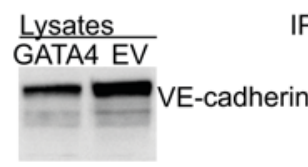

IP: VE-cadherin IgG control GATA4 EV GATA4 EV

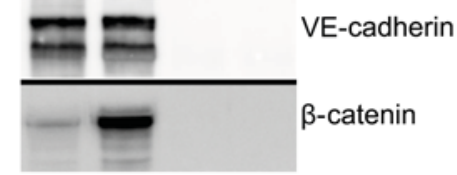

Lysates

GATA4 EV

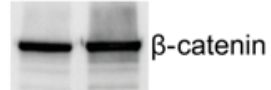

IP: $\beta$-catenin IgG control GATA4 EV GATA4EV

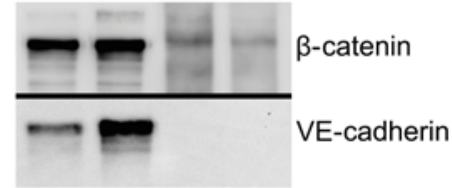

Figure 8. Endothelial GATA4 regulates VE-cadherin expression and junctional stability in liver sinusoidal and continuous ECs in vivo and in vitro. (A) IHC of VE-cadherin in the liver of Stab2-Cre Gata $4^{f / f l}$ embryos at E11.5. Scale bars: $50 \mu \mathrm{m}$ (left). Co-IF of VE-cadherin and LYVE1 in liver of Lyve1-Cre Gata4 $4^{f / f l}$ embryos at E11.5 $(n=3)$. Scale bar: $20 \mu \mathrm{m}$ (right). (B) Co-IF of VE-cadherin, TOTO-3, and phalloidin of GATA4-transduced HUVECs ( $n=3$ ). Scale bars: $60 \mu \mathrm{m}$ (left) and $30 \mu \mathrm{m}$ (right). (C) WB and densitometric quantification of VE-cadherin, $\alpha$-catenin, and $\beta$-catenin of CATA4-transduced HUVECs ( $n=4$ ). (D) Co-IP of VE-cadherin and $\beta$-catenin of GATA4-transduced HUVECs. WB of lysates is shown as control, WB of precipitates (IP) with anti-VE-cadherin, anti- $\beta$-catenin, and the respective lgG controls $(n=4)$. Student's $t$ test; ${ }^{* *} P<0.01$.

increased at E13.5 (Figure 9A), while periodic acid-Schiff (PAS) staining revealed an increase in delicate stromal fibers (Supplemental Figure 8F). Detailed analysis showed increased deposition of collagen I, III, and IV, as well as tenascin C preferentially in a perivascular location (Figure 9B). Similar changes in ECM deposition were also seen in Lyve1-Cre Gata $4^{f / f l}$ mice (Supplemental Figure 8G). In addition, increased deposition of LAMA4 and COL15A1 was seen around hepatic microvessels of Stab2Cre Gata $4^{f / f l}$ mice (Figure 9C), confirming the results of the gene expression analyses (Figure 7, A and C, and Supplemental Figure 7). Altogether, 11 ECM-associated genes were significantly upregulated in Stab2-Cre Gata $4^{f / f l}$ livers as detected by gene expression analysis, i.e., Sparcl1 (fold change [FC] 6.21), Plod2 (FC 2.55), Vcan (FC 2.28), Has2 (FC 2.28), Lama4 (FC 2.18), Col15a1 (FC 2.01), Col1a2 (FC 1.62), Cthrc1 (FC 1.57), Col4a1 (FC 1.44), Col18a1 (FC 1.37), and Col4a2 (FC 1.32). We further hypothesized that deposi- tion of altered ECM molecules in the space of Disse by Gata4-deficient LSECs might lead to activation of stellate cells. Indeed, LX2 stellate cells displayed increased adhesion on LAMA4 and collagen IV substrates in vitro (Supplemental Figure 8H).

Capillarization of LSECs impairs fetal hematopoiesis. To determine the effects of sinusoidal capillarization within the hepatic vascular niche on liver development, we investigated general parameters such as proliferation, apoptosis, hypoxia, and the major hepatic cell types in the fetal liver besides ECs. Apoptosis was significantly increased at E11.5 but not at E10.5 in Stab2-Cre Gata4 $4^{f / f l}$ livers (Figure 10A), whereas overall proliferation was unchanged (Supplemental Figure 6A). In Stab2-Cre Gata $4^{f / f l}$ mutant livers, the level of hypoxia was comparable to that in control livers at E10.5 (Supplemental Figure 6B). As the fetal liver is the major site of erythropoiesis from E13.5 onward, hemoglobin levels in the peripheral blood of Stab2-Cre Gata $4^{f / f l}$ and Lyve1-Cre Gata $4^{f / f l}$ embry- 
A Sirius red

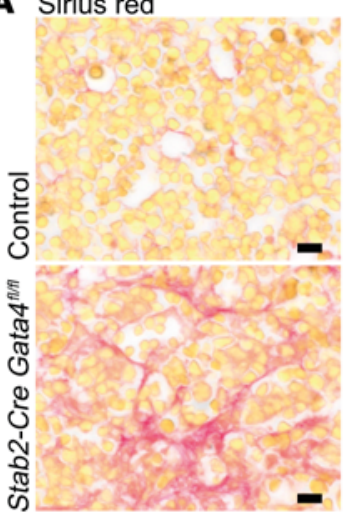

B
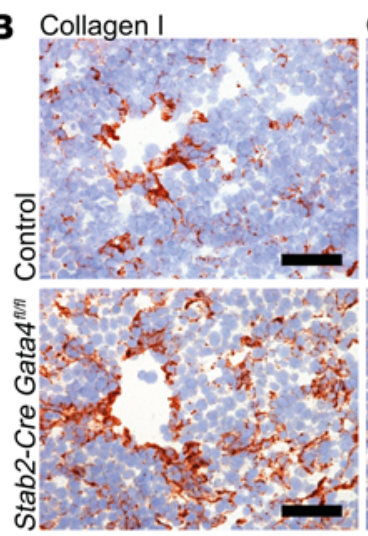

Collagen III
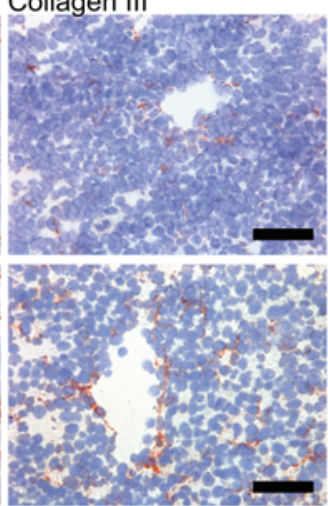

Collagen IV
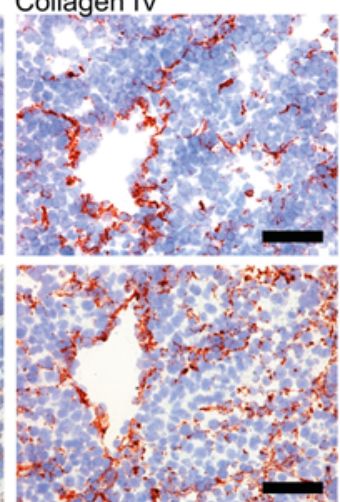

Tenascin C

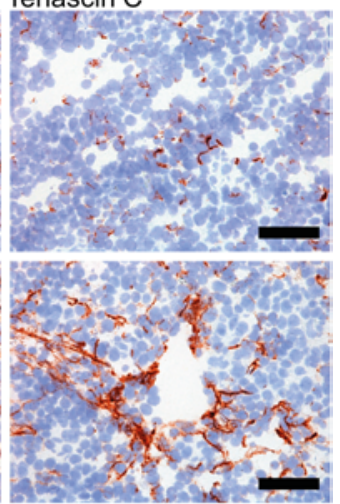

C LAMA4

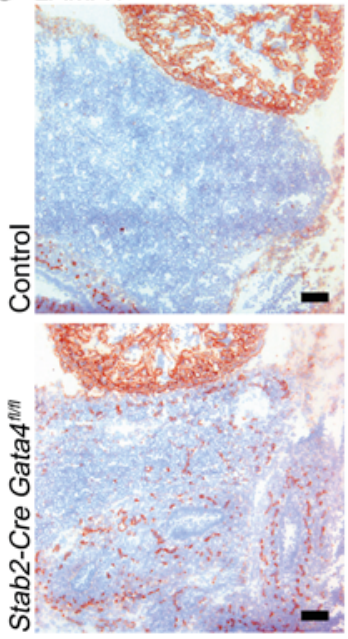

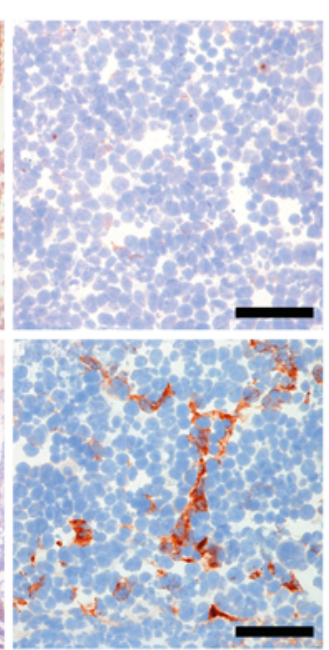
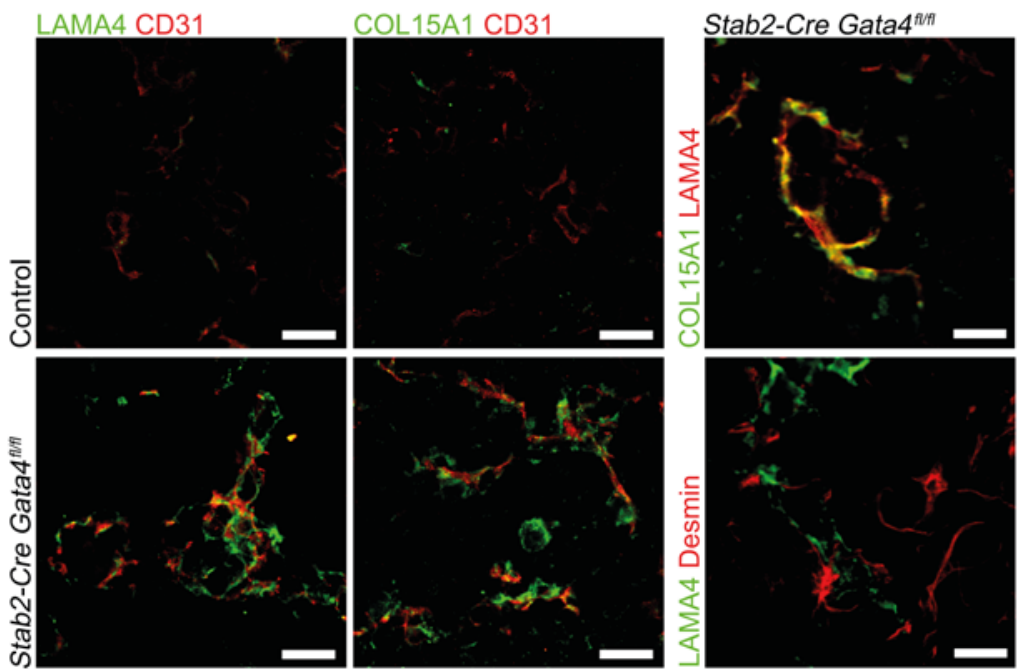

Figure 9. Liver endothelial GATA4 prevents peri-sinusoidal ECM deposition. (A) Sirius red staining in the liver of Stab2-Cre Gata fl/ff embryos at E13.5 $(n=3)$. Scale bars: $10 \mu \mathrm{m}$. (B) IHC of collagen I, collagen III, collagen IV, and tenascin C in the liver of Stab2-Cre Gata4 ${ }^{f / / f l}$ embryos at E13.5 ( $\left.n=3\right)$. Scale bars: $50 \mu \mathrm{m}$. (C) Expression of LAMA4 in the liver of Stab2-Cre Gata4fl/fl embryos at E11.5 $(n=3)$. IHC of LAMA4. Scale bars: $100 \mu \mathrm{m}$ (overview) and $50 \mu \mathrm{m}$ (magnification) (left panels). Co-IF of CD31 with LAMA4 or collagen 15a1. Scale bars: $20 \mu \mathrm{m}$ (middle panels). Co-IF of LAMA4 with COL15A1 or desmin. Scale bars: $10 \mu \mathrm{m}$ (right panels).

os were determined at E14.5 and revealed severe anemia (Figure 10B), while hemoglobin levels at E11.5 did not differ significantly from controls. This indicated that hepatic capillarization occurred well before development of fetal anemia. In addition, videos of the beating heart of E11.5 Stab2-Cre Gata $4^{f / f l}$ embryos demonstrated the presence of an intact circulation (Supplemental Video 1). These results indicate that neither anemia nor perturbation of the blood circulation is the cause of liver hypoplasia in the mutant embryos. There was no skin hemorrhage and no neck edema in E10.5E13.5 embryos (Figure 3A and Figure 5A). Relative numbers of $\mathrm{LIV}^{+}$hepatoblasts/hepatocytes and Ter119+ ${ }^{+}$erythroid cells were not significantly altered in Stab2-Cre Gata $4^{\text {fl/fl }}$ (Figure 10C) and Lyve1-Cre Gata $4^{f l / f l}$ (Supplemental Figure 5D) livers at E11.5. The numbers of desmin ${ }^{+}$stellate cells were significantly increased in Stab2-Cre Gata $4^{f / f l}$ livers at E13.5, but not at E11.5 (Figure 10D). Notably, immunofluorescence staining of fetal livers at E11.5 revealed a strong reduction in $\mathrm{CD}^{+} 8^{+}$and $\mathrm{F} 4 / 80^{+}$macrophages in Stab2-Cre Gata ${ }^{f / f l}$ animals (Figure 10E). FACS analyses of fetal liver cells confirmed the reduction in myeloid cells $\left(\mathrm{CD} 11 \mathrm{~b}^{+} \mathrm{F} 4 /\right.$ $80^{\mathrm{lo}}, \mathrm{CD} 11 \mathrm{~b}^{+} \mathrm{F} 4 / 80^{+}$) in Stab2-Cre Gata4 ${ }^{f / f l}$ mice at E11.25 (Figure
10F) and in Lyve1-Cre Gata $4^{f l / l}$ mice at E13.25 (Figure 10G). These results demonstrate conclusively that hepatic capillarization mediated by LSEC-specific deletion of Gata4 preceded impairment of the hepatic vascular niche by apoptosis and changes in cellular composition as well as development of fetal anemia.

Discontinuous LSEC specification promotes permissiveness of the hepatic vascular niche for HSC immigration. In view of the reduction in hepatic myeloid cell and macrophage numbers at E11.25 and E11.5 in Stab2-Cre Gata $4^{f / f l}$ mice (Figure 10, E and F) and at E13.25 in Lyve1-Cre Gata $4^{f / f l}$ mice (Figure 10G), we considered whether defects in hematopoiesis might aggravate malfunction of the hepatic vascular niche. Recently, it was shown that $\mathrm{CD} 45^{\text {lo }} \mathrm{Kit}^{+}$yolk sac-derived (YS-derived) erythro-myeloid progenitors (EMPs) colonizing the fetal liver around E10.5 are the founders of fetal erythropoiesis and myelopoiesis and the origin for most adult tissue-resident macrophage populations (32). In fetal livers of E11.25 Stab2-Cre Gata $4^{f / f l}$ embryos, the numbers of $\mathrm{CD} 45^{\mathrm{lo}} \mathrm{Kit}^{+}$and $\mathrm{CD} 45^{+} \mathrm{Kit}^{+/-}$cells were strongly reduced in comparison to controls (Figure 11A), as were common myeloid progenitors (CMPs) and granulocyte/macrophage progenitors 
A

TUNEL

Control

Stab2-Cre Gata4

Caspase 3

Control

Stab2-Cre Gata4
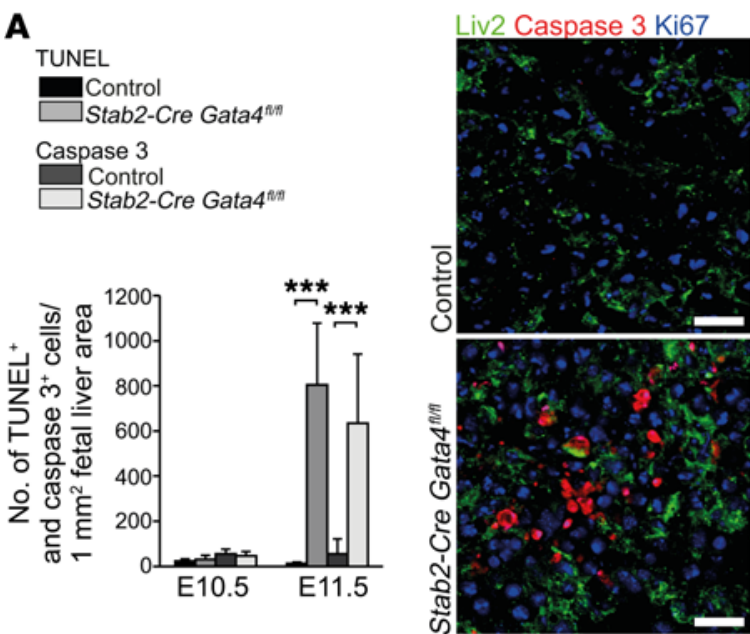

B

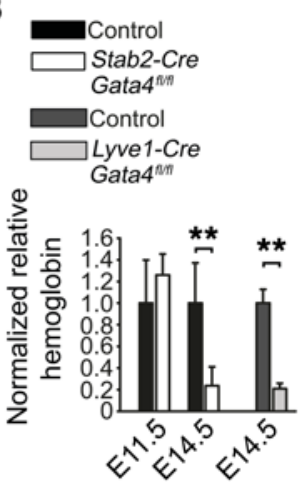

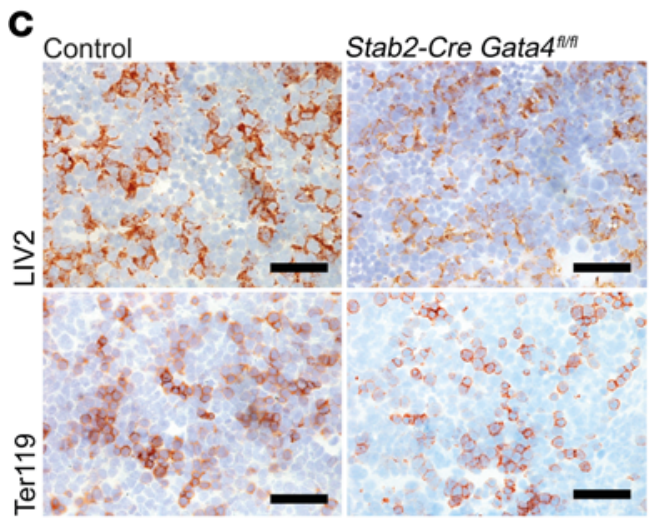

D

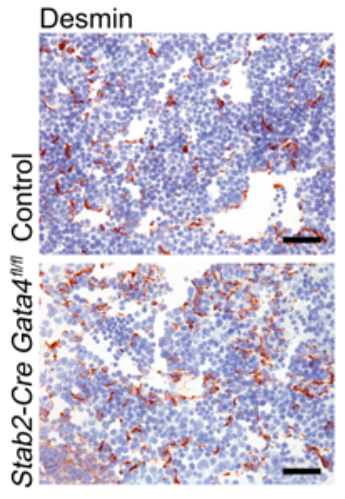

F $\underline{E} 11.25$
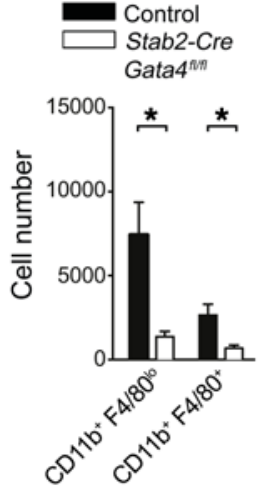
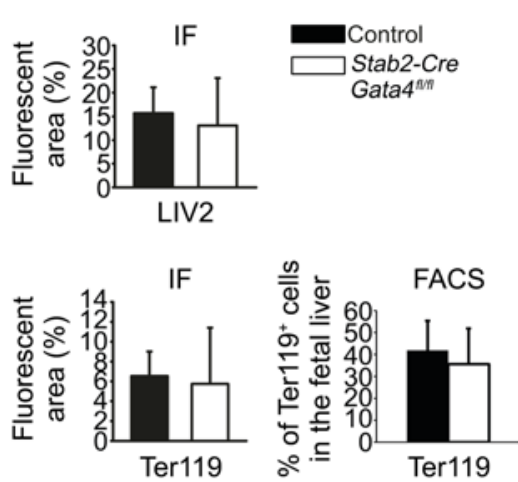

Figure 10. Liver endothelial GATA4 is required for the formation of the fetal hepatic vascular niche. (A) Quantification of TUNEL assay and IF of cleaved caspase-3 in the fetal liver at E10.5 and E11.5 $(n=3)$. Co-IF of cleaved caspase-3, Ki67, and LIV2 in the liver of Stab2-Cre Gata $4^{f / f l}$ embryos $(n=3)$. Scale bars: $20 \mu \mathrm{m}$. (B) Normalized hemoglobin levels from peripheral blood of Stab2-Cre Gata4 fl/fl embryos at E11.5 and E14.5 and of Lyve1-Cre Gata $4^{f / / f l}$ embryos at E14.5 $(n=3)$. (C) Expression of LIV2 and Ter119 in the liver of Stab2-Cre Gata $4^{\text {fl/fl }}$ embryos. IHC and quantification based on IF images at E11.5 $(n>3)$. Scale bars: 50 $\mu \mathrm{m}$. FACS quantification of Ter119+ cells in relation to total liver cells at E11.25. Data set from Figure 3D. (D) IHC of desmin in the fetal liver of Stab2-Cre Gata4 $4^{f / f 1}$ embryos at E11.5 $(n=3)$. Scale bars: $50 \mu \mathrm{m}$ (left). Quantification based on IF at E11.5 and E13.5 $(n=3)$. (E) Co-IF of LYVE1, F4/80, and CD68 in the liver of Stab2-Cre Gata $4^{f l / f l}$ embryos at E11.5 $(n=3)$. Scale bars: $20 \mu \mathrm{m}$. (F and G) Quantification upon FACS analysis of CD11b ${ }^{+} \mathrm{F} 4 / 80^{10}$ and CD11 $\mathrm{b}^{+} \mathrm{F} 4 / 80^{+}$cells in the liver of Stab2-Cre Gata4 ${ }^{f / f l}$ embryos at E11.25 (F) and in the liver of Lyve1-Cre Gata $4^{f l / f l}$ embryos at E13.25 (C) Stab2-Cre Gata4fl/fl: $n=5$ mutants and 6 controls; Lyve1-Cre Gata ${ }^{f / / f l}: n=6$. Student's $t$ test; ${ }^{*} P<0.05$, ${ }^{* *} P<0.01,{ }^{* * *} P<0.001$
E
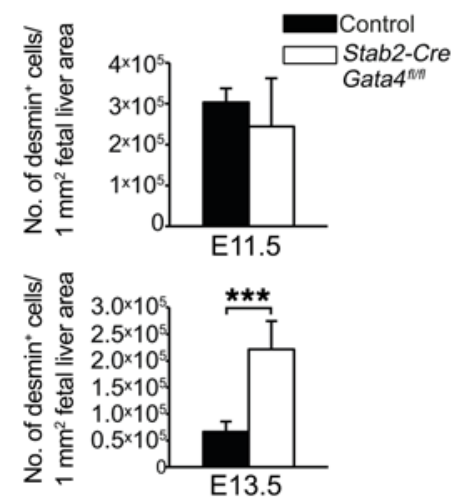

G $\underline{E} 13.25$
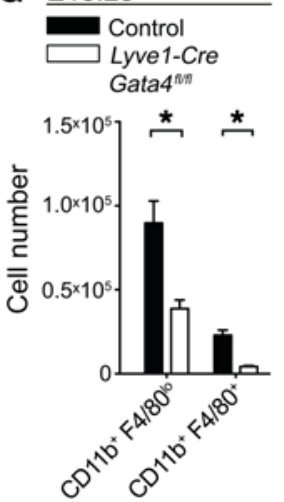
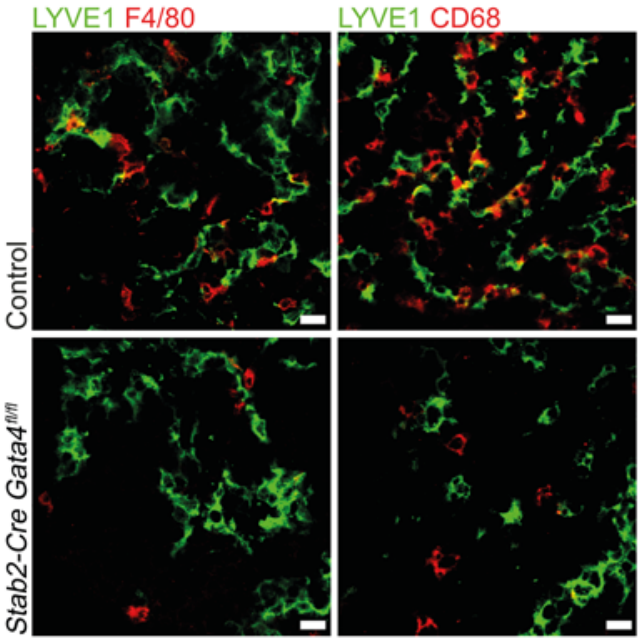
A

\section{E11.25}

\section{$\square$ Control
$\square$ Stab2-Cre Gata4t/fit}
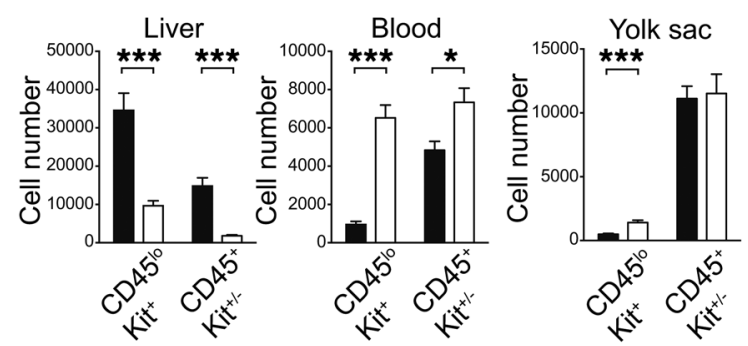

B

\section{E11.25}

Control

Stab2-Cre Gata4 ${ }^{\text {frm }}$

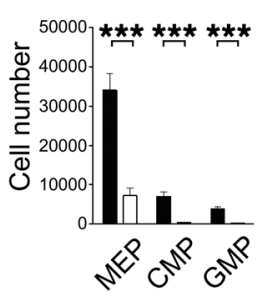

C

E13.25

$\square$ Control
$\square$ Lyve1-Cre Gata4

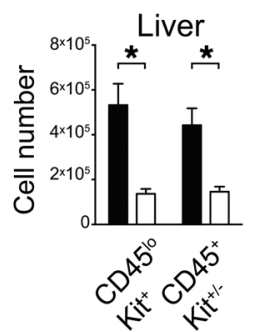

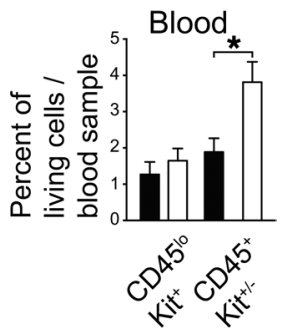

D

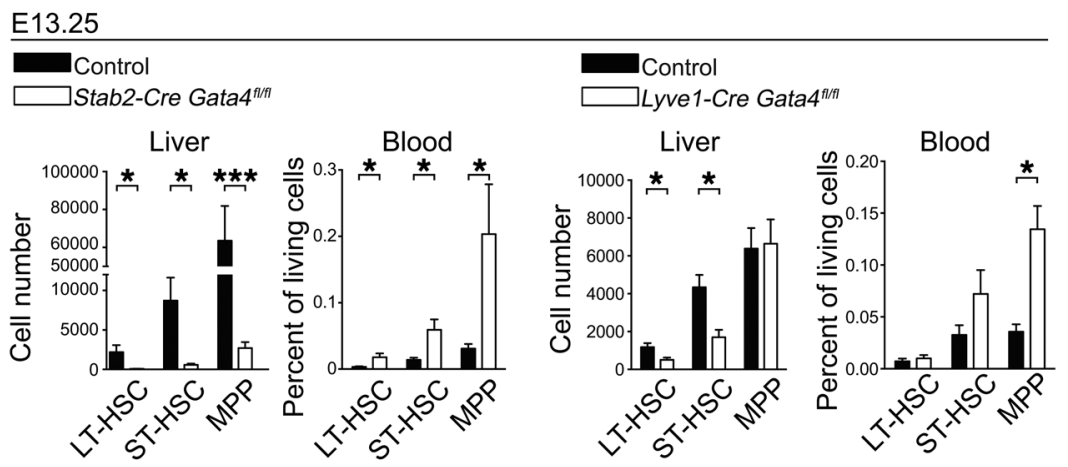

Figure 11. Liver endothelial GATA4 controls hepatic colonization by hematopoietic stem and progenitor cells. (A) FACS quantification of CD45 $5^{\circ} \mathrm{Kit}{ }^{+}$and $\mathrm{CD} 45^{+} \mathrm{Kit}^{+/-}$hematopoietic progenitors in liver, blood, and YS of Stab2-Cre Gata4 $4^{f / f l}$ embryos at E11.25 (liver: $n=14$ mutants and 10 controls; blood: $n=15$ mutants and 10 controls; YS: $n=8$ mutants and 16 controls [YS controls: Stab2-Cretg/WT Gata4f/WT, Stab2-Cre ${ }^{W T / W T}$ Cata $^{f / / f l}$, Stab2-Cre ${ }^{W T / W T}$ Gata $4^{f / W T}$ ). (B) FACS quantification of CMPs, GMPs, and myeloid-erythroid progenitors (MEP) in the liver of Stab2-Cre Gata4fl/fl embryos at E11.25 ( $n=7$ ). (C) FACS quantification of CD45 ${ }^{\circ} \mathrm{Kit}^{+}$and CD45 $\mathrm{Kit}^{+/-}$hematopoietic progenitors in the liver and blood of Lyve1-Cre Gata4 $4^{f / f l}$ embryos at E13.25 (liver: $n=6$; blood: $n=6$ mutants and 5 controls). (D) FACS quantification of long-term HSCs (LT-HSC), short-term HSCs (ST-HSC), and MPPs in liver and blood of Stab2-Cre Gata $4^{f / / f l}$ (left panels) and Lyve1-Cre Gata4fl/fl (right panels) embryos at E13.25. LT-HSCs (Lin-Sca-1+Kit+CD150+CD48-), ST-HSCs (Lin-Sca-1+Kit+CD150-CD48-), and MPPs

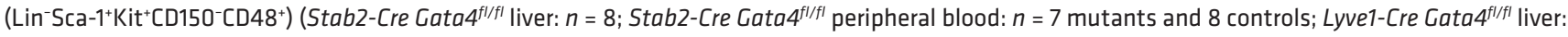
$n=6$; Lyve1-Cre Gata4 ${ }^{f / f f}$ peripheral blood: $n=6$ mutants and 5 controls). Student's $t$ test; ${ }^{*} P<0.05,{ }^{* * *} P<0.001$.

(GMPs) (Figure 11B). Notably, peripheral blood samples revealed an opposite picture, with increased numbers of $\mathrm{Kit}^{+}$cells (Figure 11A). Similarly, Lyve1-Cre Gata $4^{f / f l}$ mice showed a reduction in $\mathrm{CD} 45^{\mathrm{lo}} \mathrm{Kit}^{+}$and $\mathrm{CD} 45^{+} \mathrm{Kit}^{+/-}$cells in fetal livers and, conversely, an elevation of $\mathrm{CD} 45^{+} \mathrm{Kit}^{+/-}$cells in the peripheral blood at E13.25 (Figure 11C). In addition to EMPs, the fetal liver is also colonized by HSCs. Flow cytometric analyses of HSCs of E13.25 Stab2Cre Gata $4^{f l / f l}$ embryos showed that long-term HSCs (LT-HSCs), short-term HSCs (ST-HSCs), and multipotent progenitors (MPPs) were significantly reduced in fetal livers, but increased in the peripheral blood (Figure 11D). Similarly, Lyve1-Cre Gata4 $4^{f / f l}$ mice showed a reduction in LT- and ST-HSCs in fetal livers, but an elevation in MPPs in the peripheral blood (Figure 11D). The disturbed translocation of HSCs and progenitor cells from the circulation into the fetal liver indicated that transendothelial migration of these cells was impaired.

To confirm that the putative HSC populations from mutant and control fetal livers have full long-term multilineage potential, we performed transplantation experiments using immunodeficient $\mathrm{Rag}^{-{ }^{-/}} \gamma \mathrm{Cc}^{-/-} \mathrm{Kit}^{W / W v}$ mice as recipients (33). HSCs from Stab2-Cre Gata $4^{f / f l}$ and control mice were both able to generate lymphoid (B and $\mathrm{T}$ cells) and myeloid (granulocytes) lineages (Figure 12A). Of note, DNA analyses revealed that the recon- stituting HSC-derived populations contained recombination ${ }^{+}$ Gata4-deficient cells (Figure 12B). On CFU assays, both $\mathrm{YFP}^{+}$ Gata4-deficient as well as control HSCs were able to expand and differentiate in vitro (Figure 12C). Together, these results argue against a cell-autonomous, intrinsic defect of HSCs in Stab2-Cre Gata $4^{f l f l}$ mutant mice.

On the contrary, the reverse distribution of hematopoietic stem and progenitor cells in the peripheral blood and the livers of Stab2-Cre Gata4 $4^{f / f l}$ mice supports an endothelial-dependent dysfunction of transendothelial stem cell migration. The upregulation of VE-cadherin and VE-cadherin-associated junctional complexes in Gata4-deficient hepatic vessels as found here (Figure 8, A-D) may contribute substantially to impairment of hepatic colonization in Stab2-Cre Gata $4^{f / / l}$ mutant mice; intriguingly, Ter119+ erythroid cells seemed to be trapped in the lumina of VE-cadherin ${ }^{+}$ vessels in Stab2-Cre Gata4 $4^{f / f l}$ mice, while the lumina of most LYVE1 $^{+}$sinusoids in the control embryos lacked Ter119+ cells (Figure 13, A and B).

\section{Discussion}

The major findings described here establish that GATA4 is the molecular master regulator that orchestrates specification of discontinuous sinusoidal endothelium during liver development. 
A

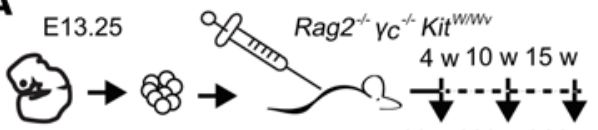

Transfer of sorted fetal liver LSK cells

Donor fetal liver
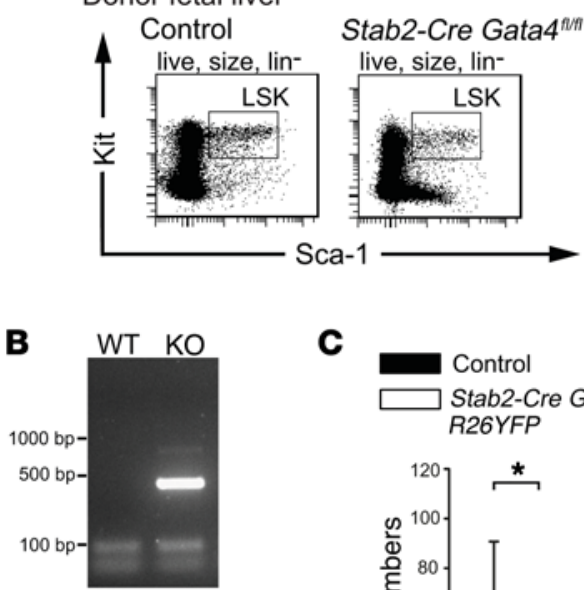

blood blood blood
Recipient blood (15 weeks)

Control Stab2-Cre Gata4 ${ }^{\text {n/m }}$

live, size, donor live, size, donor
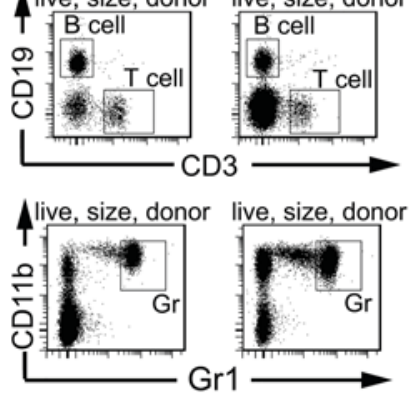

$\mathrm{Gr} 1$

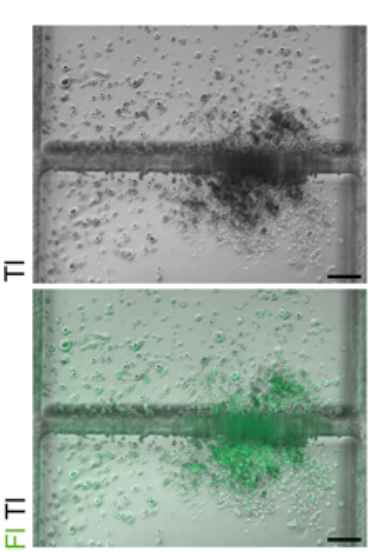

Figure 12. Analyses of the lineage potential of hematopoietic fetal liver cells from Stab2-Cre Gata4 ${ }^{f / f l}$ embryos. (A) Fetal liver donor LSK (Lin Sca- $1^{+}$Kit $\left.^{+}\right)$cells from Stab2-Cre Gata $4^{f / / f l}(n=3)$ or control $(n=5)$ embryos at E13.25 were FACS sorted and transferred into $\operatorname{Rag2}^{-/-} \gamma \mathrm{C}^{-/-} \mathrm{Kit}^{\mathrm{W/Wv}}$ mice. After 4, 10 , and 15 weeks, peripheral blood was analyzed for donor-derived lymphoid (CD3 ${ }^{+} \mathrm{T}$ cells, CD19+ $\mathrm{B}$ cells) and myeloid (CD11b+Cr1+ granulocytes) cells. Flow cytometry blots of donor fetal liver cells from control and Stab2-Cre Gata4 $4^{f / f l}$ embryo (left) as well as FACS blots of peripheral blood analysis of the corresponding recipient mouse after 15 weeks (right) are shown. (B) Product from PCR shows the recombination of floxed Gata4 allele in the blood of Stab2-Cre Gata4 $4^{\text {flfl }}$ recipient mouse after 15 weeks of transplantation. WT, Stab2-Cre ${ }^{W T / W T}$ Cata $^{f / / W T}$. (C) Numbers of CFU assay performed with fetal liver cells harvested from Stab2-Cre R26YFP Gata4 ${ }^{f / / f l}$ embryos at E12.5 ( $n=4$ mutants and 7 controls) (left). Representative image shows a fluorescent colony from the mutant embryo (right). TI, transmission light; FI, fluorescent light. Scale bars: $200 \mu \mathrm{m}$. Student's $t$ test; ${ }^{*} P<0.05$.
This GATA4-dependent transcriptional program determines the functional competence of the hepatic sinusoidal endothelium. As suggested by the fact that the gene expression signatures of fetal LSECs and ectopically GATA4-expressing cultured continuous ECs only partially overlap, organ-specific vascular functions differ in a context-dependent manner: (a) liver endothelial-associated angiokines such as WNT2, HGF, RSPO3, ANG2, and BMP2 do not seem to play a major role in liver development, while they do in establishing metabolic zonation in adult liver $(18,19)$ or in regulating adult liver regeneration $(10,11)$; (b) GATA4-expressing LSECs are permissive for transmigration of HSCs during early fetal development, while they become non-permissive during later fetal development despite continued expression of transcription factor GATA4 into adulthood. Notably, metabolic liver zonation only develops well after birth and is paralleled by development of angiodiversity within the liver microvasculature into centrilobular/central vein, midlobular, and periportal segments $(15,16,18$, 19). Furthermore, the developmental and molecular hierarchies that generate angiodiversity in all the different segments of the hepatic vascular trees other than LSECs remain to be elucidated.

As GATA4 did not suffice to fully convert generic continuous ECs to bona fide LSECs and vice versa, other transcriptional regulators or extravascular cues may contribute to specification of LSECs and may thereby modify the basic transcriptional program of LSECs in order to dynamically adapt hepatic angiodiversity to the different contextual needs during development, maturation, and adulthood, and in health and disease. Regarding
GATA-binding proteins, Fog2-deficient embryos die at mid-gestation due to complex cardiac defects, including tetralogy of Fallot, while other malformations such as underdevelopment of the lungs and smaller liver size were reported to be secondary to cardiac failure (34). In addition, when FOG2 expression was rescued by transgenic overexpression specifically in cardiomyocytes, the resultant embryos were grossly normal, confirming that lung and liver pathology in Fog2-deficient mice was secondary to cardiac failure (34). Previously, we have identified Lmo3, Tfec, and Maf as further LSEC-associated transcription factors (25). While Maf awaits further study, Lmo3-deficient mice were reported to survive into adulthood and do not show any gross abnormalities (35). Tfec has recently been shown to be an endothelial transcription factor involved in shaping the fetal hematopoietic stem cell niche in zebrafish, the so-called caudal hematopoietic tissue (CHT) (36). CHT, however, does not correspond to the developing liver in mammals. Endothelial Notch signaling also contributes to LSEC differentiation and seems to be essential for the development and maintenance of a proper hepatic vascular architecture and its function (37). Functionally, sinusoidal dilatation and enhanced angiogenesis as seen in endothelial Notch signaling-deficient LSECs may promote hepatic metastasis (38). Notably, sinusoidal dilatation is a common end point of several signaling defects in LSECs; for example, plexin B2-transgenic mice defective in Rho guanine nucleotide exchange factor binding show sinusoidal dilatation and further abnormalities in the architecture of the liver vasculature (39). Recently, LXR $\alpha$ has 
A
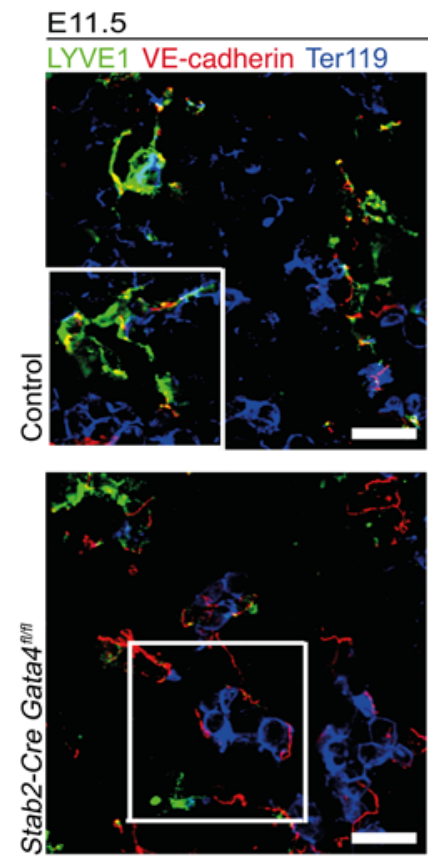
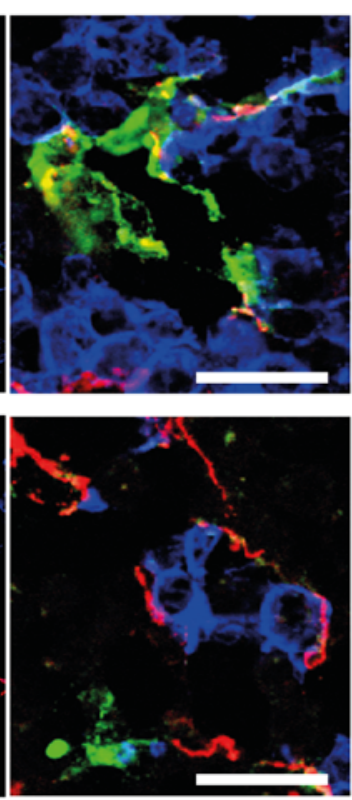

B

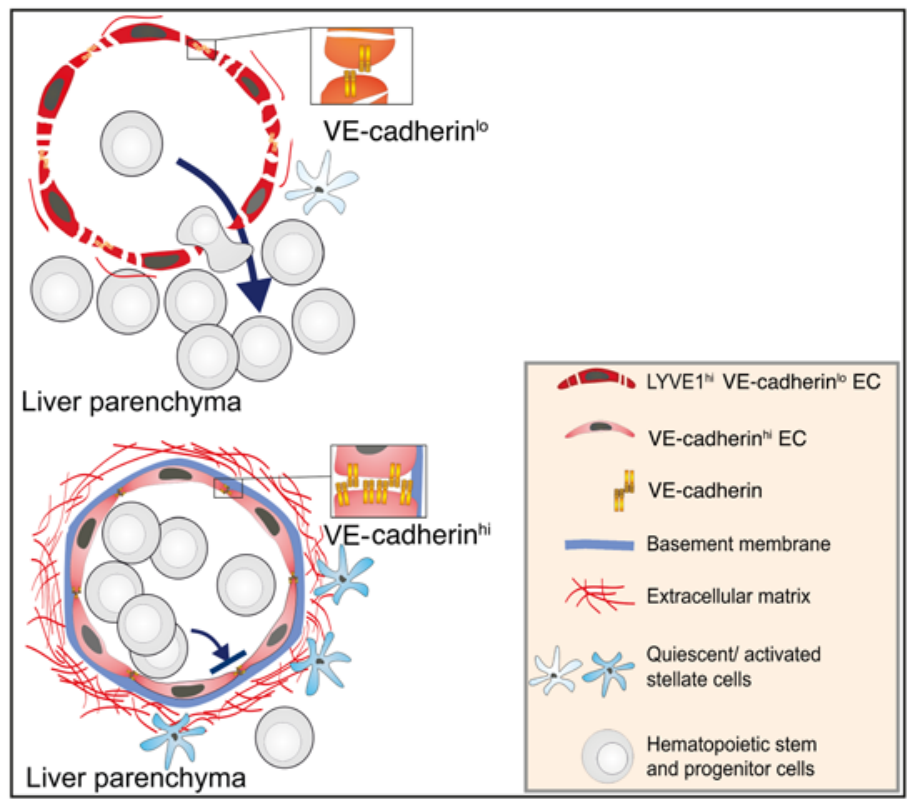

Figure 13. Endothelial GATA4 expression preserves liver sinusoidal endothelial identity with high junctional permeability and limited ECM deposition permissive for hepatic stem cell colonization and unperturbed liver development and erythropoiesis. (A) Co-IF of LYVE1, Ter119, and VE-cadherin in the liver of Stab2-Cre Gata ${ }^{f / / f l}$ embryos at E11.5 $(n=3)$. Scale bars: $20 \mu \mathrm{m}$. (B) GATA4 serves as the master regulator of the fetal hepatic vascular niche. During normal development, GATA4-dependent gene expression cascades in ECs promote discontinuous sinusoidal endothelial differentiation featuring weak cell-cell contacts, lack of a basement membrane, and low levels of ECM deposition, resulting in an antifibrotic perivascular microenvironment. The hepatic sinusoidal microvasculature supports transmigration of hematopoietic stem and progenitor cells into the liver parenchyma, a process indispensable for liver development. In the absence of GATA4, hepatic sinusoidal microvessels undergo continuous transdifferentiation/capillarization, including basement membrane formation followed by ECM deposition and stellate cell activation, and lethal impairment of hematopoiesis via stabilization of VE-cadherin+ junctions.

been shown to prevent Hedgehog signaling in LSECs and subsequent development of LSEC capillarization in a pathological setting of adult hepatic fibrosis (40). While these latter signaling pathways all contribute to the proper differentiation and function of the hepatic microvasculature during adulthood, the findings presented here identify transcription factor GATA4 as a superordinate molecular regulator of hepatic angiodiversity controlling development. GATA4 acts as an indispensable relay that promotes organ-specific sinusoidal endothelial differentiation by repression of continuous EC specification.

Impaired HSC immigration into mutant fetal livers demonstrates that formation of a functional hematopoietic stem cell niche in the fetal liver depends on liver endothelial expression of GATA4. Notably, it has been shown that artificial junctional tightening by overexpression of a VE-cadherin/ $\alpha$-catenin fusion protein in EC impaired immigration into the nascent hematopoietic stem cell niche in the liver in a very similar manner $(41,42)$. Thus, low-level endothelial junctional stability is an indispensable requirement for HSC immigration into the fetal liver. As GATA4 suppresses VE-cadherin expression and the abundance of VE-cadherin/ $\beta$-catenin complexes in ECs, it can be assumed that GATA4 is indeed the physiologic regulator of low-level junctional stability in fetal LSECs that controls permissiveness for HSCs. As the superordinate regulation of hepatic angiodiversity by GATA4 is coupled to a downstream complexity of developmental interactions in the hepatic vascular niche and the liver as a whole, it remains an open question whether other angiocrine factors of fetal LSECs including the composition of the perivascular ECM are also involved in establishing the hematopoietic stem cell niche of the fetal liver. In this respect, studies in zebrafish have suggested that cytokines such as stem cell factor/kitlg may be involved (36). Notably, exit from the liver microvascular hematopoietic stem cell niche is mediated by adhesive properties of PLVAP expressed by LSECs between E11.5 and E15.5 (43), while it is mediated by changes in pericyte content postnatally. After closure of the umbilical inlet at birth, portal vessels undergo a transition from a NRP1 $1^{+} \mathrm{EPHB} 2^{+}$artery to an $\mathrm{EPHB} 4^{+}$vein phenotype associated with loss of periportal nestin $^{+} \mathrm{NG2}^{+}$pericytes that allows emigration of hematopoietic stem cells from the liver (17).

In general, the results presented here provide a framework for understanding the molecular mechanisms and functions of microvascular angiodiversity in the liver and other organs. By defining the molecular program that governs hepatic angiodiversity and by defining its role in shaping the architecture and function of the hepatic vascular niche in fetal hematopoiesis, our results support the concept that angiocrine factors not only comprise angiokines, i.e., endothelial secreted growth factors, but a variety of other instructive cues produced by ECs, such as morphogens, chemokines, cytokines, ECM components, and exosomes, as well as membrane-bound factors such as adhesion molecules; furthermore, they confirm that angiocrine 
factors may be produced constitutively or may be modulated during development, regeneration, and disease processes (7).

Furthermore, our findings may have important clinical ramifications. Future work will be needed to address whether molecular manipulation of angiodiversity by GATA4 can be applied to improve in vitro differentiation of organ-specific endothelium (44) and generation of bio-artificial liver organoids (45). In vivo, sinusoidal GATA4 expression was shown to be considerably decreased in human advanced liver fibrosis (29). Furthermore, GATA4 expression was also reduced in isolated LSECs from a rat cirrhotic liver model (46). As reprogramming of myofibroblasts into hepatocytes by a combination of transcription factors has already been successfully demonstrated to improve liver fibrosis $(47,48)$, prevention and treatment of sinusoidal capillarization by GATA4-mediated reprogramming may soon become reality $(49,50)$. It has recently been shown that single-wall carbon nanotubes are highly promising vehicles for pharmaceutical interventions that preferentially target the hepatic microvasculature via sinusoidal endothelial scavenger receptors STAB1 and STAB2 (51). Therefore, angiodiversity-targeted therapies may prove beneficial not only in the treatment of fibrotic liver diseases, but more generally in diseases such as diabetes or cancer that are accompanied by microvascular transdifferentiation.

\section{Methods}

Detailed methods are described in Supplemental Methods.

Generation of Stab2-Cre mice. A Cre recombinase cDNA was fused into exon 1 of stab2 via homologous recombination in a BAC clone. Linearized DNA was injected into the pronuclei of fertilized C57BL/ 6NCRL oocytes (see Supplemental Methods). The transgenic founder B6.Stabilin- $2^{\text {tg1.2cre }}$ was used as the stabilin 2-Cre (Stab2-Cre) line.

Immunostaining, LacZ staining, electron microscopy, and routine histology. Fetal and adult tissues were harvested, processed, stained, and analyzed according to standard protocols (see Supplemental Methods for protocols and antibodies used).

Apoptosis assays. Embryo sections $(2 \mu \mathrm{m})$ were stained for cleaved caspase-3 and were used for TUNEL assay according to the manufacturer's protocol (Merck Millipore).

Hypoxyprobe measurements. Oxygen supply to mouse embryos was visualized by the Hypoxyprobe-1 method on 2- $\mu$ m PFA-fixed, paraffin-embedded sections according to the manufacturer's protocol (Hypoxyprobe) (see Supplemental Methods).

Fetal hemoglobin measurement. Blood taken from E11.5 and E14.5 embryos was analyzed based on the photometric detection of cyanmethemoglobin.

Western blot and ELISA. Western blotting was carried out as described in Supplemental Methods. BMP-2 in cell culture supernatants of transduced HUVECs was analyzed with the DuoSet Elisa kit (R\&D Systems).

Quantitative RT-PCR. Relative gene expression in relation to the reference gene ( $\beta$-actin) was quantified by quantitative RT-PCR (qRT-PCR) experiments as described previously (see Supplemental Methods).

Microarray processing and statistical analysis. Gene expression profiling was performed using arrays Mogene-2.0-st and HG-U133 Plus 2.0-st from Affymetrix. The raw and normalized data were deposited in the NCBI's Gene Expression Omnibus database (GEO GSE20375 and GSE92357).
Adhesion assays. Adhesion of Lx-2 cells (provided by Scott Friedman, Mount Sinai Hospital, New York, New York, USA) was quantified either by crystal violet staining of cells adhering to 96-well plates coated with collagen IV (Sigma-Aldrich), LN-211, or LN-411 (BioLamina) or by a real-time cell analyzer xCELLigence system (Roche) (see Supplemental Methods).

FACS. For flow cytometric analysis, YS, liver, and blood cells were harvested from embryos (E11.25 and E13.25). To obtain single cells, tissue was digested (YS), mechanically dissociated (liver), or only washed with PBS/FCS (blood). Fc receptors were blocked by incubating cells with purified mouse IgG $(500 \mathrm{mg} / \mathrm{ml}$, Jackson ImmunoResearch Laboratories Inc.) and stained with commercially prepared antibodies (see Supplemental Methods). Cells were analyzed on a FACSFortessa (BD), and data were analyzed using FACSDiva software (BD).

Statistics. Statistical analysis was performed with SigmaPlot 11.0 Software (Systat Software). For comparisons, 2-tailed Student's $t$ test or 1-way ANOVA was used. Differences between data sets with $P<0.05$ were considered statistically significant. Data are presented as means, with error bars indicating standard deviation. Controls for each experiment are described in Supplemental Methods.

Study approval. All animal studies were approved by the animal ethics committee in Baden-Wuerttemberg, Regierungspraesidium Karlsruhe, Karlsruhe, Germany.

\section{Author contributions}

CG, PSK, KS, and SG designed the experiments. CG, PSK, JZ, $\mathrm{KK}, \mathrm{KB}, \mathrm{VO}, \mathrm{TL}, \mathrm{AD}, \mathrm{FU}, \mathrm{MD}$, SS, CS, KBH, KR, BA, and KS performed experiments and acquired data. CG, PSK, JZ, KK, KB, VO, TL, AD, FD, MD, SS, CS, KBH, KR, BA, HGA, KS, and SG analyzed data. $\mathrm{KBH}, \mathrm{SMK}, \mathrm{TP}$, and $\mathrm{HRR}$ provided reagents. $\mathrm{CG}$ and SG wrote the manuscript draft. All authors reviewed and edited the manuscript.

\section{Acknowledgments}

We thank Günter Küblbeck, Monica Adrian, Siladitta Biswas, Jochen Weber, Hiltrud Schönhaber, Jeanine Bahadori, Sven Schäfer, Maria Muciek, Carolina De La Torre, Michaela Plößer, Andrea Homburger, and Alexandra Müller for excellent technical support. We also thank Michelle Neßling for help with the generation of electron microscopic images (Core Facility Electron microscopy, DKFZ Heidelberg). We are grateful to Martin Leverkus (Rheinisch-Westfälische Technische Hochschule [RWTH] Aachen) and Moritz Felcht (Medical Faculty Mannheim) for scientific discussions. We acknowledge the support of the Core Facility Live Cell Imaging Mannheim at the CBTM (DFG INST 91027/9-1 FUGG). This work was supported in part by grants from the Deutsche Forschungsgemeinschaft (DFG) SFB-TRR23, project B1 (to CG and SG); SFBTRR77, project C3 (to SG); SFB 938, project H (to SG); GRK2099/ RTG2099, project 7 (to CG and SG); and by grants from the Academy of Finland \#284605 and \#259872 (to SMK and TP).

Address correspondence to: Cyrill Géraud, Department of Dermatology, Venereology, and Allergology, University Medical Center and Medical Faculty Mannheim, Theodor-Kutzer-Ufer 1-3, 68163 Mannheim, Germany. Phone: 00.496.213.832.617; E-mail: cyrill.geraud@umm.de. 
1. Aird WC. Phenotypic heterogeneity of the endothelium: I. Structure, function, and mechanisms. Circ Res. 2007;100(2):158-173.

2. Nolan DJ, et al. Molecular signatures of tissue-specific microvascular endothelial cell heterogeneity in organ maintenance and regeneration. Dev Cell. 2013;26(2):204-219.

3. Aird WC. Phenotypic heterogeneity of the endothelium: II. Representative vascular beds. Circ Res. 2007;100(2):174-190.

4. Lawson ND, et al. Notch signaling is required for arterial-venous differentiation during embryonic vascular development. Development 2001;128(19):3675-3683.

5. You LR, Lin FJ, Lee CT, DeMayo FJ, Tsai MJ, Tsai SY. Suppression of Notch signalling by the COUP-TFII transcription factor regulates vein identity. Nature. 2005;435(7038):98-104.

6. Wigle JT, Oliver G. Prox1 function is required for the development of the murine lymphatic system. Cell.1999;98(6):769-778.

7. Rafii S, Butler JM, Ding BS. Angiocrine functions of organ-specific endothelial cells. Nature. 2016;529(7586):316-325.

8. Cao Z, et al. Angiocrine factors deployed by tumor vascular niche induce B cell lymphoma invasiveness and chemoresistance. Cancer Cell. 2014;25(3):350-365.

9. Cao Z, et al. Targeting of the pulmonary capillary vascular niche promotes lung alveolar repair and ameliorates fibrosis. Nat Med. 2016;22(2):154-162.

10. Ding BS, et al. Divergent angiocrine signals from vascular niche balance liver regeneration and fibrosis. Nature. 2014;505(7481):97-102.

11. $\mathrm{Hu}$ J, et al. Endothelial cell-derived angiopoietin- 2 controls liver regeneration as a spatiotemporal rheostat. Science. 2014;343(6169):416-419.

12. Itkin $\mathrm{T}$, et al. Distinct bone marrow blood vessels differentially regulate haematopoiesis. Nature. 2016;532(7599):323-328.

13. Géraud C, et al. Unique cell type-specific junctional complexes in vascular endothelium of human and rat liver sinusoids. PLoS One. 2012;7(4):e34206.

14. Schledzewski K, et al. Deficiency of liver sinusoidal scavenger receptors stabilin- 1 and -2 in mice causes glomerulofibrotic nephropathy via impaired hepatic clearance of noxious blood factors. JClin Invest. 2011;121(2):703-714.

15. Walter TJ, Cast AE, Huppert KA, Huppert SS. Epithelial VEGF signaling is required in the mouse liver for proper sinusoid endothelial cell identity and hepatocyte zonation in vivo. Am J Physiol Gastrointest Liver Physiol. 2014;306(10):G849-G862.

16. Xie G, Wang L, Wang X, Wang L, DeLeve LD. Isolation of periportal, midlobular, and centrilobular rat liver sinusoidal endothelial cells enables study of zonated drug toxicity. Am J Physiol Gastrointest Liver Physiol. 2010;299(5):G1204-G1210.

17. Khan JA, et al. Fetal liver hematopoietic stem cell niches associate with portal vessels. Science. 2016;351(6269):176-180.
18. Rocha AS, et al. The Angiocrine factor Rspondin3 is a key determinant of liver zonation. Cell Rep. 2015;13(9):1757-1764.

19. Wang B, Zhao L, Fish M, Logan CY, Nusse R. Self-renewing diploid Axin2(+) cells fuel homeostatic renewal of the liver. Nature. 2015;524(7564):180-185.

20. DeLeve LD. Liver sinusoidal endothelial cells in hepatic fibrosis. Hepatology. 2015;61(5):1740-1746

21. Xie G, et al. Role of differentiation of liver sinusoidal endothelial cells in progression and regression of hepatic fibrosis in rats. Gastroenterology. 2012;142(4):918-927.e6.

22. LeCouter J, et al. Angiogenesis-independent endothelial protection of liver: role of VEGFR-1. Science. 2003;299(5608):890-893.

23. Lammert E, Cleaver O, Melton D. Induction of pancreatic differentiation by signals from blood vessels. Science. 2001;294(5542):564-567.

24. Matsumoto K, Yoshitomi H, Rossant J, Zaret KS. Liver organogenesis promoted by endothelial cells prior to vascular function. Science. 2001;294(5542):559-563.

25. Géraud C, et al. Liver sinusoidal endothelium: a microenvironment-dependent differentiation program in rat including the novel junctional protein liver endothelial differentiation-associated protein-1. Hepatology. 2010;52(1):313-326.

26. Rivera-Feliciano J, et al. Development of heart valves requires Gata 4 expression in endothelial-derived cells. Development. 2006;133(18):3607-3618.

27. Sato $Y$, et al. Notch mediates the segmental specification of angioblasts in somites and their directed migration toward the dorsal aorta in avian embryos. Dev Cell. 2008;14(6):890-901.

28. Crosswhite PL, et al. CHD4-regulated plasmin activation impacts lymphovenous hemostasis and hepatic vascular integrity. JClin Invest. 2016;126(6):2254-2266.

29. Delgado I, et al. GATA4 loss in the septum transversum mesenchyme promotes liver fibrosis in mice. Hepatology. 2014;59(6):2358-2370.

30. Nonaka H, Tanaka M, Suzuki K, Miyajima A. Development of murine hepatic sinusoidal endothelial cells characterized by the expression of hyaluronan receptors. Dev Dyn. 2007;236(8):2258-2267.

31. Zheng R, et al. Function of GATA factors in the adult mouse liver. PLoS One. 2013;8(12):e83723.

32. Gomez Perdiguero E, et al. Tissue-resident macrophages originate from yolk-sac-derived erythro-myeloid progenitors. Nature. 2015;518(7540):547-551.

33. Waskow C, Madan V, Bartels S, Costa C, Blasig R, Rodewald HR. Hematopoietic stem cell transplantation without irradiation. Nat Methods. 2009;6(4):267-269.

34. Tevosian SG, et al. FOG-2, a cofactor for GATA transcription factors, is essential for heart morphogenesis and development of coronary vessels from epicardium. Cell. 2000;101(7):729-739.

35. Tse E, et al. Null mutation of the Lmo4 gene or a combined null mutation of the Lmo1/Lmo3 genes causes perinatal lethality, and Lmo 4 controls neural tube development in mice. Mol Cell Biol. 2004;24(5):2063-2073.

36. Mahony CB, Fish RJ, Pasche C, Bertrand JY. tfec controls the hematopoietic stem cell vascular niche during zebrafish embryogenesis. Blood. 2016;128(10):1336-1345.

37. Cuervo H, Nielsen CM, Simonetto DA, Ferrell L, Shah VH, Wang RA. Endothelial notch signaling is essential to prevent hepatic vascular malformations in mice. Hepatology. 2016;64(4):1302-1316.

38. Banerjee $\mathrm{D}$, et al. Notch suppresses angiogenesis and progression of hepatic metastases. Cancer Res. 2015;75(8):1592-1602.

39. Worzfeld T, et al. Genetic dissection of plexin signaling in vivo. Proc Natl Acad Sci U S A. 2014;111(6):2194-2199.

40. Xing Y, Zhao T, Gao X, Wu Y. Liver X receptor $\alpha$ is essential for the capillarization of liver sinusoidal endothelial cells in liver injury. Sci Rep. 2016;6:21309.

41. Wessel F, et al. Leukocyte extravasation and vascular permeability are each controlled in vivo by different tyrosine residues of VE-cadherin. Nat Immunol. 2014;15(3):223-230.

42. Dartsch N, Schulte D, Hägerling R, Kiefer F, Vestweber D. Fusing VE-cadherin to $\alpha$-catenin impairs fetal liver hematopoiesis and lymph but not blood vessel formation. Mol Cell Biol. 2014;34(9):1634-1648.

43. Rantakari P, et al. Fetal liver endothelium regulates the seeding of tissue-resident macrophages. Nature. 2016;538(7625):392-396

44. Ginsberg M, et al. Efficient direct reprogramming of mature amniotic cells into endothelial cells by ETS factors and TGF $\beta$ suppression. Cell. 2012;151(3):559-575.

45. Takebe $\mathrm{T}$, et al. Vascularized and functional human liver from an iPSC-derived organ bud transplant. Nature. 2013;499(7459):481-484.

46. Tugues S, et al. Microarray analysis of endothelial differentially expressed genes in liver of cirrhotic rats. Gastroenterology. 2005;129(5):1686-1695.

47. Song $\mathrm{G}$, et al. Direct reprogramming of hepatic myofibroblasts into hepatocytes in vivo attenuates liver fibrosis. Cell Stem Cell. 2016;18(6):797-808.

48. Rezvani M, et al. In vivo hepatic reprogramming of myofibroblasts with AAV vectors as a therapeutic strategy for liver fibrosis. Cell Stem Cell. 2016;18(6):809-816.

49. Wong WT, Cooke JP. Therapeutic transdifferentiation of human fibroblasts into endothelial cells using forced expression of lineagespecific transcription factors. J Tissue Eng. 2016;7:2041731416628329.

50. Ginsberg M, Schachterle W, Shido K, Rafii S. Direct conversion of human amniotic cells into endothelial cells without transitioning through a pluripotent state. Nat Protoc. 2015;10(12):1975-1985.

51. Alidori S, et al. Deconvoluting hepatic processing of carbon nanotubes. Nat Commun. 2016;7:12343. 